\title{
$\begin{array}{ll}\text { Research Square } & \text { Preprints are preliminary reports that have not undergone peer review. } \\ \text { They should not be considered conclusive, used to inform clinical practice, }\end{array}$

\section{Prognostic and Immunological Role of mRNA ac4C Regulator NAT10 in Pan-Cancer: New Territory for Cancer Research?}

\section{Chuanxi Yang}

Medical school of southeast university

\section{Tingting Wu}

The first affiliated hospital of nanjing medical university

Jing Zhang

The first affiliated hospital of nanjing medical university

Jinghui Liu

The first affiliated hospital of nanjing medical university

\section{Kun Zhao}

The first affiliated hospital of nanjing medical university

\section{Wei Sun}

The first affiliated hospital of nanjing medical university

\section{Jing Shi}

The first affiliated hospital of nanjing medical university

\section{Xiangqing Kong ( $\nabla$ xiangqing_kong@sina.com )}

The first affiliated hospital of nanjing medical university

\section{Research}

Keywords: NAT10, prognosis, pan-cancer, tumor infiltration, N4-acetylcytidine

Posted Date: October 21st, 2020

DOI: https://doi.org/10.21203/rs.3.rs-94029/v1

License: (1) (1) This work is licensed under a Creative Commons Attribution 4.0 International License. Read Full License

Version of Record: A version of this preprint was published at Frontiers in Oncology on May 19th, 2021. See the published version at https://doi.org/10.3389/fonc.2021.630417. 


\section{Abstract}

\section{Background}

NAT10 (also known as human N-acetyltransferase-like protein) is a critical gene that regulates N4acetylcytidine formation in RNA, similar to the multiple regulators of N6-methyladenosine. However, the underlying functions and mechanisms of NAT10 in tumor progression and immunology are unclear.

\section{Methods}

In this study, we systematically analyzed the pan-cancer expression and correlations of NAT10, using databases including Oncomine, PrognoScan, GEPIA, and Kaplan-Meier Plotter. The potential correlations of NAT10 with immune infiltration stages and gene marker sets were analyzed using the Tumor Immune Estimation Resource and GEPIA.

\section{Results}

Compared with normal tissues, NAT10 showed higher expression in 26 of 27 cancers based on combined data from TCGA and GTEx. In different datasets, high NAT10 expression was significantly correlated with poor prognosis in adrenocortical carcinoma, head and neck squamous cell carcinoma, liver hepatocellular carcinoma, kidney renal papillary cell carcinoma, and pheochromocytoma and paraganglioma. Moreover, there were significant positive correlations between NAT10 expression and immune infiltrates, including B cells, CD8+ T cells, CD4+ T cells, neutrophils, macrophages, dendritic cells, endothelial cells, and fibroblasts in LIHC. NAT10 expression showed strong correlations with diverse immune marker gene sets in LIHC.

Conclusion

NAT10 expression affects the prognosis of pan-cancer patients and is significantly correlated with tumor immune infiltration. Furthermore, it represents a potential target for cancer therapy.

\section{Background}

RNA modification was first discovered in 1956 by Cohn et al. (1) and Davis et al. (2). In recent years, extensive research in RNA biology has revealed diverse modifications of RNA at the post-transcription stage. More than 100 RNA modifications have been shown to have important roles in regulating RNA stability (3), localization (4), transport, shearing (5), and translation (6). In particular, N6-methyladenosine (m6A), the most abundant mRNA modification in eukaryotic cells, with its regulators have been a focus of attention in recent years because of their extensive participation in various physiological and pathological process, including development $(7,8)$, immunity $(9)$, tumorigenesis and metastasis $(10)$, stem cell renewal (11), and adipogenesis (12). However, the roles of other RNA modifications in tumors have not been identified, and the targeting regulatory proteins for all the modifications are yet to be discovered and confirmed. 
N4-acetylcytidine (ac4C) is considered to be a conservative chemically modified nucleoside on tRNA and rRNA (13). Recently, several studies proved that the presence of ac4C on tRNA, rRNA and mRNA is important for increasing and maintaining the fidelity of protein translation (14-17). Furthermore, studies by Thomale et al. (18) and Liebich et al. (19) found significant increases in modified nucleosides (including ac4C) in the urine of tumor mice and cancer patients. Besides, increased levels of ac4C in urine were observed in colorectal cancer (20), urogenital cancer (21), ovarian epithelial cancer (22), and breast cancer (23). These findings suggest that ac4C is a potential biomarker for cancer.

NAT10 (also known as hALP, human N-acetyltransferase-like protein), which was first reported in 2003, is a protein with histone acetylation activity that can enhance telomerase activity by stimulating transcription of hTERT (24). Tuan et al. first showed that NAT10 was associated with cancer by demonstrating that it could significantly promote cell growth in epithelial ovarian cancer (25). NAT10 also has a potential role in increasing melanogenesis and melanoma growth (26). In addition, high NAT10 expression was found to be related to poor survival in human hepatocellular carcinoma (27) and to promote colorectal cancer progression by increasing micronuclei (28). These findings suggest that NAT10 has multifaceted functional roles in cancers. Several studies have also shown that levels of ac4C are associated with inflammatory responses $(29,30)$. However, the underlying functions and mechanisms of NAT10 in tumor progression and tumor immunology remain unclear.

In the current study, we systematically analyzed the pan-cancer expression of NAT10 and its correlations, using databases including Oncomine, PrognoScan, GEPIA, and Kaplan-Meier Plotter. We then investigated the potential correlations of NAT10 with immune infiltration stages using the Tumor Immune Estimation Resource (TIMER) and GEPIA. The findings from our study indicate that NAT10 expression affects the prognosis of pan-cancer patients as well as being significantly correlated with tumor-immune infiltration. Furthermore, it may serve as a potential target for cancer therapies.

\section{Results}

\subsection{Pan-cancer NAT10 mRNA expression levels}

The mRNA expression levels of NAT10 were analyzed in Oncomine over a cancer-wide range. NAT10 expression was higher in cancer groups compared with the respective normal groups, including bladder, breast, colorectal, esophageal, gastric, liver, lung, kidney, and prostate cancers, as well as leukemia and myeloma. Interestingly, lower expression of NAT10 was found in one leukemia dataset (Fig. 1A). The NAT10 expression data for multiple cancers from Oncomine are summarized in Supplementary Table 1.

The GTEx database was also used to examine different tissues from healthy individuals; the mRNA expression levels of NAT10 were similar across all tissues (Fig. 1D), with the notable exceptions of bone marrow and blood. More significantly, in different cancer cell lines from the CCLE database, not only were NAT10 expression levels widely increased, but there was a narrower range of expression compared with that in normal tissues. (Fig. 1E). Furthermore, the pan-cancer expression of NAT10 was examined based on RNA sequencing data from TCGA using TIMER. Details of expression in tumor tissues and adjacent 
normal tissues are shown in Fig. 1B. NAT10 expression was significantly increased in 17 of 23 cancer types. Based on combined data from TCGA and GTEx, the expression of NAT10 was significantly elevated in 26 of 27 cancers (Fig. 1C).

\subsection{Analysis of the pan-cancer link between NAT10 expression and multifaceted prognostic value}

We assessed the correlation between the respective expression levels of NAT10 and OS, PFS, DFS, and DSS in different cancer types using a single-variate Cox regression analysis based on TCGA. The results are summarized in Fig. 2A-D. Nine of the 33 cancer types showed significant relationships between NAT10 expression levels and OS, seven showed significant relationships with PFS, five with DFS, and seven with DSS. Overall, the HRs for NAT10 were significant for LIHC, HNSC, ACC, KIRP, and PCPG with respect to OS, PFS, DFS, and DSS. In addition, survival curves comparing high and low expression of NAT10 in different types of cancer in the TCGA database were shown in supplementary figure 1.

Using Kaplan-Meier Plotter and GEPIA, high expression of NAT10 in HNSC, KIRP, LIHC and PCPG had worse outcomes from Kaplan-Meier Plotter in OS and RFS (Fig. 3A-H). For ACC, HNSC, KIRP and LIHC, NAT10 significantly decreased the OS in GEPIA (Fig. 3I-M). In addition, compared with low expression levels, high expression levels of NAT10 were correlated with poorer DFS in ACC, KIRP and LIHC in GEPIA (Fig. 3N-P). Using PrognoScan, we analyzed the role of NAT10 in each cancer type (number of cancer types $=12$ ) and the relationships between NAT10 expression and prognosis in different cancers. The results are shown in Supplementary Table 2. Therefore, these results suggest that NAT10 expression is an independent risk factor for poor prognosis in these cancers.

\subsection{High NAT10 expression affects the prognosis of LIHC with different clinicopathological features}

In order to determine the relevance and underlying mechanisms of NAT10 expression in LIHC, we first analyzed NAT10 expression at different stages of LIHC, ACC, KIRP, and HNSC using TIMER. The expression of NAT10 at stage III showed a significant increase compared with stage I (Fig. 4A-D). The relationships between NAT10 expression and clinicopathological features were investigated by combining clinical and pathological data in Kaplan-Meier Plotter. With respect to OS and PFS, almost all characteristics showed a detrimental role of NAT10 in patients with LIHC, except for grade $2(\mathrm{~N}=174, \mathrm{HR}$ $=1.92,95 \% \mathrm{Cl}=0.97$ to $3.97, \mathrm{P}=0.0564), \mathrm{AJCC}_{-} \mathrm{T} 1(\mathrm{~N}=180, \mathrm{HR}=1.6,95 \% \mathrm{Cl}=0.89$ to $2.89, \mathrm{P}=0.1146)$, and micro-vascular invasion $(\mathrm{N}=90, \mathrm{HR}=2.02,95 \% \mathrm{Cl}=0.9$ to $4.57, \mathrm{P}=0.0833)$ for OS; and stage $2(\mathrm{~N}=$ $84, \mathrm{HR}=1.87,95 \% \mathrm{Cl}=0.99$ to $3.54, \mathrm{P}=0.0501)$, grade $2(\mathrm{~N}=175, \mathrm{HR}=1.51,95 \% \mathrm{Cl}=0.98$ to $2.35, \mathrm{P}=$ $0.0619)$, non-vascular invasion ( $N=204, \mathrm{HR}=1.54,95 \% \mathrm{Cl}=0.96$ to $2.49, \mathrm{P}=0.0721)$, and microvascular invasion $(\mathrm{N}=91, \mathrm{HR}=1.76,95 \% \mathrm{Cl}=0.97$ to $3.19, \mathrm{P}=0.0583$ ) for PFS (Supplementary Table 3). Therefore, the expression of NAT10 seems to be an independent risk factor in prognosis of LIHC.

\subsection{NAT10 expression is correlated with pan-cancer immune infiltration levels}

Previous studies have proved that tumor-infiltrating lymphocytes can affect patient survival (31), and the above results demonstrate a powerful pan-cancer effect of NAT10 on prognosis. Thus, we explored the 
relationships between inflammatory infiltration and NAT10 expression. Using TIMER datasets, we calculated the coefficients of NAT10 expression and immune infiltration levels in 40 cancer types. The results show that NAT10 expression has significant positive correlations with tumor purity in 15 types of cancer. In addition, NAT10 expression had significant correlations with infiltrating levels of B cells in 15 types of cancer, CD8+ T cells in 17 types of cancer, CD4+ T cells in 20 types of cancer, macrophages in 13 types of cancer, neutrophils in 23 types of cancer, and DCs in 19 types of cancer (Supplementary Table 4).

To investigate the distinct types of cancers in which NAT10 was associated with prognosis and immune infiltration, and considering that tumor purity influences the analysis of immune infiltration, we first assessed the relationships between NAT10 expression and tumor purity in the above five types of cancer. Two types (ACC and HNSC) of the five showed significant positive correlations with tumor purity in TIMER. In addition, consistent positive correlations with different types of infiltrating immune cells were seen in LIHC: neutrophils $(R=0.162, P=0.009)$ and $D C s(R=0.129, P=0.039)$ in KIRP; $B$ cells $(R=0.243$, $P=0.002)$ and macrophages $(R=0.221, P=0.004)$ in PCPG; $B$ cells, $C D 4+T$ cells, neutrophils, and $D C s$ in ACC; and CD8+ T cells, neutrophils and DCs in HNSC showed positive correlations with NAT10 expression (Fig. 5A-E). These findings strongly suggest that NAT10 affects patient survival via interactions with immune cell infiltration in cancers including LIHC.

\subsection{Relationships between NAT10 expression and immune markers}

To further investigate the correlations between NAT10 and different types of infiltrating immune cells, we analyzed the relationships between NAT10 and immune cell markers using TIMER and GEPIA. In TIMER, after adjustments for tumor purity, NAT10 expression was significantly associated with 42 of 45 immune cell markers in LIHC; however, it was significantly correlated with only 22 gene markers in KIRP, eight gene markers in ACC, 28 gene markers in HNSC and 21 gene markers in PCPG (Table 1).

As shown in Fig. 5, B cells, CD4+ T cells, and macrophages were the three immune cell types most strongly correlated with NAT10 expression in LIHC. However, these correlations were not found in KIRP. The relationships between NAT10 expression and B cells, CD4+ T cells, and macrophage markers also showed differences between LIHC and KIRP. First, as for B cells and macrophage markers, we analyzed the correlations of NAT10 expression in tumor and normal tissues for LIHC and KIRP based on the GEPIA database. Notably, the correlations between NAT10 and TAMs were similar to those found using TIMER, suggesting that NAT10 is correlated with TAM infiltration in LIHC. Second, NAT10 expression in LIHC and KIRP showed partial difference in its relationships with CD8+ T cells, Tfh cells, Th2 cells, Th9 cells, Th17 cells, Th22 cells, neutrophils, and NK cells. In addition, NAT10 in LIHC had significant correlations with T cell exhaustion markers including PD-1 and CTLA4, and monocyte markers including CD14 and CD16, whereas NAT10 in KIRP showed no such relationships. We also used MCPcounter datasets to analyze the correlations between NAT10 expression and other immune cells; the results, shown in Supplementary Fig. 2 , revealed strong positive correlations of endothelial cells and fibroblasts with NAT10 expression in KIRP 
and LIHC. Therefore, these results further confirm the findings that NAT10 is specifically correlated with immune infiltrating cells in LIHC, demonstrating that NAT10 has a vital role in immune escape in LIHC.

\subsection{Pan-cancer correlation of NAT10 expression with expression of immune checkpoint genes}

Tumor immunotherapy is a novel treatment that involves restarting and maintaining the tumor-immune cycle to restore the body's normal anti-tumor immune response. Immune checkpoint genes are the main direction for monoclonal antibody inhibitors, cancer vaccines, cell therapies, and small-molecule inhibitors (32). Thus, we analyzed the relationships between NAT10 expression and 47 immune checkpoint genes in the above five types of cancer. Figure 6 shows the most significant positive correlations in KIRP (15 of 47) and LIHC (31 of 47); no such strong relationships were found in HNSC (three of 47), ACC (three of 47), or PCPG (seven of 47), but there were positive correlations. Therefore, these results further suggest that NAT10 expression has a vital role related to immune checkpoint genes in KIRP and LIHC (Fig. 6A).

\subsection{Relationships between NAT10 expression and immune neoantigens, TMB and MSI}

Neoantigens are new unnatural proteins encoded by mutated genes in tumor cells, which can be used to synthesize new antigen vaccines to activate immunity and achieve a therapeutic effect (33). Hence, we counted the number of new antigens in the above five types of cancer and analyzed the relationships between NAT10 expression and these antigens. The results are shown in Fig. 6B. Surprisingly, there was no relationship between NAT10 expression and antigens.

Tumor mutation load (or TMB) (34), a quantifiable biomarker used to reflect the number of mutations contained in tumor cells, and MSI (35), the emergence of a new microsatellite allele in the tumor, are valid prognostic biomarkers and indicators of immune therapy response in many tumor types. Therefore, we analyzed the correlations of NAT10 expression with TMB and MSI in the above five types of cancer, using Person correlation. As shown in Fig. 6C, NAT10 expression was positively correlated with low TMB in KIRP $(P=0.0061)$. In addition, the coefficient values for MSI indicated that NAT10 expression is positively correlated with high MSI in LIHC (P = 0.0092, Fig. 6D). Overall, these results show that the relationships of NAT10 expression with TMB and MSI are diverse among these five types of cancer.

\subsection{Interactions and correlations of predicted proteins with NAT10 in LIHC}

NAT10, the only confirmed regulator of mRNA acetyltransferase, shows remarkable correlation with LIHC. However, as in the case of m6A RNA methylation regulators which change the levels of m6A, the details of the compounds involved in the acetylation of mRNA are not clear. To further explore the mechanism of NAT10 in liver cancer, STRING tools were used to predict the proteins interacting with NAT10 (Fig. 7A); these included NOL10 (nucleolar protein 10, NOL10), HEATR1 (HEAT repeat-containing protein 1), BMS1 (ribosome biogenesis protein), TBL3 (transducin beta-like protein 3), WDR46 (WD repeat-containing protein 46), NOL6 (nucleolar protein 6), IMP4 (U3 small nucleolar ribonucleoprotein protein IMP4), UTP20 (small subunit processome component 20 homolog), NOP14 (nucleolar protein 14), and UTP18 (U3 small 
nucleolar RNA-associated protein 18 homolog). The interaction network was further supported by the correlation analysis in LIHC. Interestingly, NAT10 expression was strongly positively correlated with the 10 predicted genes (Fig. 7B). Furthermore, relationships between prognosis and expression of the 10 genes were investigated in LIHC with respect to OS and RFS. As shown in Supplementary Table 5, in almost all cases, higher expression of the 10 genes was associated with poor prognosis in LIHC patients, similar to NAT10. Therefore, it is reasonable to conclude that the interactions between NAT10 and these 10 genes influence the prognosis of $\mathrm{LIHC}$ patients.

\section{Materials And Methods}

\subsection{Data mining for NAT10 in public databases}

First, to investigate the pan-cancer differential expression of NAT10 mRNA, several databases were mined, including: Oncomine (http://www.oncomine.org/resource/login.html) with thresholds of P-value 0.05 and fold change 1.5; The Cancer Genome Atlas (TCGA); the Broad Institute Cancer Cell Line Encyclopedia (CCLE); and GEPIA (http://gepia.cancer-pku.cn/).

\subsection{Survival analysis in GEPIA, PrognoScan, and Kaplan-Meier Plotter}

Cox regression analysis was performed to test the correlations between NAT10 expression and patients' overall survival (OS), disease-free survival (DFS), disease-specific survival (DSS), and progression-free survival (PFS) in each cancer type using TCGA in the R environment. PrognoScan (http://dna00.bio.kyutech.ac.jp/PrognoScan/index.html) microarray datasets were used to examine the relationships of NAT10 expression levels with prognosis. The threshold was adjusted to Cox P-value < 0.05. GEPIA, an interactive online platform with information from TCGA and GTEx, was used to assess the effects of NAT10 expression on OS and DFS in each available cancer type (total number $=34$ ). Kaplan-Meier Plotter is a relatively comprehensive online tool that can be used to analyze the effects of 54,675 genes on survival in 21 cancer types. We analyzed the relationships of NAT10 with OS and relapse-free survival (RFS) in liver hepatocellular carcinoma (LIHC), Head and neck squamous cell carcinoma (HNSC), adrenocortical carcinoma (ACC), kidney renal papillary cell carcinoma (KIRP), and pheochromocytoma and paraganglioma (PCPG). Hazard ratios (HRs) with 95\% confidence intervals (Cls) and log-rank P-values were calculated.

\subsection{Correlation between NAT10 expression and immune status in TIMER and GEPIA}

TIMER, a powerful online platform for the systematic analysis of immune infiltration in abundant cancer types, contains 10,897 samples spanning 32 cancer types from the TCGA database, which can be used to evaluate the diversity of immune infiltration. Therefore, we analyzed NAT10 expression with all six types of immune infiltrates: B cells, CD4+ T cells, CD8+ T cells, neutrophils, macrophages, and dendritic cells (DCs). Correlations between expression levels of NAT10 and tumor purity were also analyzed. 
Furthermore, the correlations between immune cell markers and NAT10 expression were identified using correlation modules. The gene markers included markers of B cells, CD8+ T cells, follicular helper T cells (Tfh), T-helper 1 (Th1) cells, T-helper 2 (Th2) cells, T-helper 9 (Th9) cells, T-helper 17 (Th17) cells, T-helper 22 (Th22) cells, regulatory T cells (Tregs), exhausted T cells, M1 macrophages, M2 macrophages, tumorassociated macrophages (TAMs), monocytes, natural killer (NK) cells, neutrophils, and DCs. Correlation scores were calculated for LIHC, HNSC, ACC, KIRP, and PCPG using the Spearman method.

\subsection{PPI network construction and correlation analysis}

To explore the proteins potentially interacting with NAT10, the STRING database (http://string-db.org) was used to infer protein-protein interactions (PPIs). Pearson correlation analysis was used to determine the associations of proteins with NAT10.

\subsection{Statistical analysis}

Low and high NAT10 expression groups were established using normalized NAT10 mRNA expression values from the various datasets, based on P-values determined by t-tests. The Spearman correlation test was used to assess the correlations between NAT10 expression and targets of interest, including neoantigens, tumor mutational burden (TMB), and microsatellite instability (MSI). We used log-rank tests to calculate HRs and log-rank P-values in Kaplan-Meier Plotter, PrognoScan, and GEPIA. P-values less than 0.05 were considered significant. All graphs were produced using the R software (version 4.0.2, www.r-project.org) with the ggplot2 and forestplot packages.

\section{Discussion}

NAT10 was the first acetylation regulator to be proved to maintain effective translation and stabilize mRNA by forming ac4C on mRNA (17). Although studies of NAT10 have been limited, increased levels of ac4C in urine are known to be correlated with four types of cancer (20-23). In addition, several studies have shown that overexpression of NAT10 could promote tumor progression in cancers including colorectal cancer, epithelial ovarian cancer, and melanoma $(25,26,29)$. Here, we report that a higher level of NAT10 was comprehensively found in multiple cancers according to several different databases. Meanwhile, using OS, PFS, DFS, and DSS data from TCGA, we discovered that three of 33 cancers (ACC, $\mathrm{KIRP}, \mathrm{LIHC}$ ) showed consistent correlations between unfavorable prognosis wtih NAT10 expression; NAT10 expression in HNSC and PCPG showed significant correlations with OS, PFS, and DSS but not DFS. in particular, there were significant correlations with NAT10 expression in LIHC and KIRP. Prognosis can vary according to characteristics such as gender, race, tumor grade, and tumor stage. First, we found significant increased NAT10 expression at stage III compared with stage I in LICH, KIRP, and ACC. Second, high levels of NAT10 expression were shown to be almost consistently correlated with poor prognosis in liver cancer across gender, race, alcohol consumption, hepatitis virus, tumor stage, tumor grade and AJCC_T, with the highest HRs for poor OS and PFS. In KIRP and HNSC, these correlations were found in our study. Together, these findings strongly suggest that NAT10 represents an independent prognostic biomarker for liver cancer. 
The tumor microenvironment (TME) contains various cells including a large proportion of infiltrating immune cells (36). Conventionally, the infiltration of immune cells in the TME is a component of an antitumor strategy to avoid tumor cells being killed $(32,37)$. Furthermore, in two (ACC and HNSC) of the above five types of cancer, NAT10 expression showed a significant positive correlation with tumor purity in TIMER and a significant correlation with prognosis in GEPIA, whereas KIRP, LIHC, and PCPG had no correlation of NAT10 with tumor purity in TIMER. In addition, the types of infiltrating immune cells in five types of cancer were as follows: $B$ cells, $C D 8+T$ cells, $C D 4+T$ cells, macrophages, neutrophils, and DCs in LIHC; B cells, CD4+ T cells, neutrophils, and DCs in ACC; CD8+ T cells, neutrophils, and DCs in HNSC; neutrophils and DCs in KIRP; $B$ cells and macrophages in PCPG. Moreover, the relationships between NAT10 expression and immune cell markers reveal the role of NAT10 in regulating tumor immunology in the above five cancers. In particular, NAT10 expression was significantly associated with 42 of 45 immune cell markers in LIHC, that is, all markers except MRC1 for M2 macrophages, KIR3DL1 for NK cells, and MPO for neutrophils. However, only 20 gene markers in PCPG, 22 gene markers in KIRP, 28 gene markers in HNSC, and eight gene markers in ACC showed significant correlations with high NAT10 expression. Interestingly, most gene markers in these five types of cancer were involved in B cell, $\mathrm{T}$ cell exhaustion, and TAM. Otherwise, endothelial cells and fibroblasts were strongly positively correlated with NAT10 expression in KIRP and LIHC, whereas moderate or weak correlations with NAT10 expression were found in ACC, HNSC, and PCPG using MCPcounter datasets. These results reveal that NAT10 plays an important part in recruitment and regulation of immune infiltrating cells in LIHC.

Systematic analysis of the correlations between NAT10 expression and immune checkpoint genes (32), immune neoantigens (33), TMB (34), and MSI (35) is conducive to a more comprehensive understanding of TME, which could be used to synthesize new antigen vaccines for antitumor therapies. First, in our study of immune checkpoint genes, we found the most significant positive correlations with NAT10 expression in KIRP (15 of 47) and LIHC (31 of 47), whereas HNSC (three of 47), ACC (three of 47), and PCPG (seven of 47) did not show these strong relationships, although there were still positive correlations. Second, no relationship was found between NAT10 expression and antigens. Third, NAT10 expression was positively correlated with low TMB in KIRP $(P=0.0061)$, and with high MSI in LIHC ( $P$ $=0.0092)$. Overall, these results show that the relationships of NAT10 expression with TMB and MSI are diverse in these five types of cancer.

STRING tools showed that 10 genes (NOL10, HEATR1, BMS1, TBL3, WDR46, NOL6, IMP4, UTP20, NOP14, and UTP18) were strongly positively correlated with NAT10. Furthermore, our results show that poor prognosis with respect to OS and RFS was associated with expression of these 10 genes in LIHC. Therefore, it is reasonable to believe that the interactions between NAT10 and these 10 genes could influence the prognosis of $\mathrm{LIHC}$ patients. Recent studies have raised concerns about the function of NAT10 (38). However, there were limitations to our study. First, systematic bias may have been generated because of the large proportion of microarray and sequencing data used in our study; higher-resolution methods such as single-cell RNA sequencing could be used to overcome this issue in future studies. Second, although NAT10 plays an important part in ac4C formation in mRNA, there was no information in the databases about the detailed changes in ac4C in these cancers. Third, this study only conducted a 
bioinformatics analysis of NAT10 expression and patient survival across several databases; further experiments in vivo and in vitro should be performed in future studies. Fourth, we used STRING tools to predict the 10 genes most strongly correlated with NAT10, but future prospective studies focusing on the precise genes that interact with NAT10 in cancer could help to provide a definitive answer to the question of the function of NAT10.

\section{Conclusion}

In summary, increased NAT10 expression was correlated with poor prognosis in 12 types of cancer, especially ACC, KIRP, LIHC, HNSC, and PCPG, and with increased immune infiltration levels of CD8+ T cells, CD4+ T cells, macrophages, neutrophils, and DCs in various cancers. In addition, NAT10 expression may contribute to regulation of TAMs, B cells, exhausted T cells, and other immune cells in LIHC. Therefore, NAT10 is likely to have an independent role in immune cell infiltration and could represent a unique prognostic biomarker in patients with liver cancers.

\section{Abbreviations}

ACC, adrenalcortical carcinoma; BLCA, bladder urothelial carcinoma; BRCA, breast invasive carcinoma; CCLE, Cancer Cell Line Encyclopedia; CHOL, cholangiocarcinoma; COAD, colon adenocarcinoma; DFI, disease-free interval; DLBC, lymphoid neoplasm diffuse large B-cell lymphoma; DSS, disease-specific survival; ESCA, esophageal carcinoma; GBM, glioblastoma multiforme; GTEx, The Genotype-Tissue Expression; International Cancer Genome Consortium; HNSC, Head and Neck squamous cell carcinoma; $\mathrm{KICH}$, kidney chromophobe; KIRC, kidney renal clear cell carcinoma; KIRP, kidney renal papillary cell carcinoma; LAML, acute myeloid leukemia; LGG, brain lower grade glioma; LIHC, liver hepatocellular carcinoma; LUAD, lung adenocarcinoma; LUSC, lung squamous cell carcinoma; MESO, mesothelioma; MSI, microsatellite instability; OS, overall survival; OV, ovarian serous cystadenocarcinoma; PAAD, pancreatic adenocarcinoma; PCPG, pheochromocytoma and paraganglioma; PD-1, programmed cell death protein 1s; PRAD, prostate adenocarcinoma; READ, rectum adenocarcinoma; ROC, Receive Operating Characteristic; SARC, sarcoma; SKCM, skin cutaneous melanoma; STAD, stomach adenocarcinoma; TCGA, The Cancer Genome Atlas; TGCT, testicular germ cell tumor; THYM, thymoma; TIMER, Tumor IMmune Estimation Resource; TMB, tumor mutation burden; UCEC, uterine corpus endometrial carcinoma; UVM, uveal melanoma.

\section{Declarations}

\section{Ethics approval}

All the data included in the analysis are from public databases without the need of permissions from local ethical committees.

\section{Consent for publication}


All authors have seen and approved the manuscript and consent publication.

\section{Data availability statement}

Publicly available datasets were analyzed in our study; the data can be accessed at:

https://portal.gdc.cancer.gov/, http://www.oncomine.org/resource/login.html, http://dna00.

bio.kyutech.ac.jp/PrognoScan/index.html, https://kmplot. com/analysis/, and http://gepia.cancerpku.cn, http://cistrome.org/ TIMER/.

\section{Competing interests}

The authors declare no conflict of interest.

\section{Funding}

Supported by the Youth Program of National Natural Science Foundation of China (Grant No. 81800313) and the Youth Program of Natural Science Foundation of Jiangsu Province (Grant No. BK20181084).

\section{Authors' contributions}

$\mathrm{CY}$ and TW designed this study. JZ, JL KZ, and WS extracted the information from the databases. CY and JZ analyzed the data. XK and JS supervised the entire study. CY and TW wrote the manuscript. All authors revised and approved the final manuscript.

\section{Acknowledgements}

Not applicable

\section{References}

1. Cohn WE. Pseudouridine, a carbon-carbon linked ribonucleoside in ribonucleic acids: isolation, structure, and chemical characteristics. J Biol Chem. 1960;235:1488-98.

2. Davis FF, Allen FW. Ribonucleic acids from yeast which contain a fifth nucleotide. J Biol Chem. 1957;227(2):907-15.

3. Wang X, Lu Z, Gomez A, Hon GC, Yue Y, Han D, et al. N6-methyladenosine-dependent regulation of messenger RNA stability. Nature. 2014;505(7481):117-20.

4. Fustin JM, Doi M, Yamaguchi Y, Hida H, Nishimura S, Yoshida M, et al. RNA-methylation-dependent RNA processing controls the speed of the circadian clock. Cell. 2013;155(4):793-806.

5. Molinie B, Wang J, Lim KS, Hillebrand R, Lu ZX, Van Wittenberghe N, et al. m(6)A-LAIC-seq reveals the census and complexity of the m(6)A epitranscriptome. Nat Methods. 2016;13(8):692-8.

6. Meyer KD, Patil DP, Zhou J, Zinoviev A, Skabkin MA, Elemento O, et al. 5' UTR m(6)A Promotes CapIndependent Translation. Cell. 2015;163(4):999-1010. 
7. Zheng G, Dahl JA, Niu Y, Fedorcsak P, Huang CM, Li CJ, et al. ALKBH5 is a mammalian RNA demethylase that impacts RNA metabolism and mouse fertility. Mol Cell. 2013;49(1):18-29.

8. Yang Y, Huang W, Huang JT, Shen F, Xiong J, Yuan EF, et al. Increased N6-methyladenosine in Human Sperm RNA as a Risk Factor for Asthenozoospermia. Sci Rep. 2016;6:24345.

9. Li HB, Tong J, Zhu S, Batista PJ, Duffy EE, Zhao J, et al. m(6)A mRNA methylation controls T cell homeostasis by targeting the IL-7/STAT5/SOCS pathways. Nature. 2017;548(7667):338-42.

10. Ma JZ, Yang F, Zhou CC, Liu F, Yuan JH, Wang F, et al. METTL14 suppresses the metastatic potential of hepatocellular carcinoma by modulating N(6) -methyladenosine-dependent primary MicroRNA processing. Hepatology. 2017;65(2):529-43.

11. Zhang S, Zhao BS, Zhou A, Lin K, Zheng S, Lu Z, et al. m(6)A Demethylase ALKBH5 Maintains Tumorigenicity of Glioblastoma Stem-like Cells by Sustaining FOXM1 Expression and Cell Proliferation Program. Cancer Cell. 2017;31(4):591-606 e6.

12. Wang X, Wu R, Liu Y, Zhao Y, Bi Z, Yao Y, et al. m(6)A mRNA methylation controls autophagy and adipogenesis by targeting Atg5 and Atg7. Autophagy. 2020;16(7):1221-35.

13. Zachau HG, Dutting D, Feldmann H. The structures of two serine transfer ribonucleic acids. Hoppe Seylers Z Physiol Chem. 1966;347(4):212-35.

14. Kawai G, Hashizume T, Miyazawa T, McCloskey JA, Yokoyama S. Conformational characteristics of 4-acetylcytidine found in tRNA. Nucleic Acids Symp Ser. 1989(21):61-2.

15. Sharma S, Langhendries JL, Watzinger P, Kotter P, Entian KD, Lafontaine DL. Yeast Kre33 and human NAT10 are conserved 18S rRNA cytosine acetyltransferases that modify tRNAs assisted by the adaptor Tan1/THUMPD1. Nucleic Acids Res. 2015;43(4):2242-58.

16. Arango D, Sturgill D, Alhusaini N, Dillman AA, Sweet TJ, Hanson G, et al. Acetylation of Cytidine in mRNA Promotes Translation Efficiency. Cell. 2018;175(7):1872-86 e24.

17. Dominissini D, Rechavi G. N(4)-acetylation of Cytidine in mRNA by NAT10 Regulates Stability and Translation. Cell. 2018;175(7):1725-7.

18. Thomale J, Nass G. Elevated urinary excretion of RNA catabolites as an early signal of tumor development in mice. Cancer Lett. 1982;15(2):149-59.

19. Liebich HM, Lehmann R, Xu G, Wahl HG, Haring HU. Application of capillary electrophoresis in clinical chemistry: the clinical value of urinary modified nucleosides. J Chromatogr B Biomed Sci Appl. 2000;745(1):189-96.

20. Feng B, Zheng MH, Zheng YF, Lu AG, Li JW, Wang ML, et al. [Application of urinary nucleosides in the diagnosis and surgical monitoring of colorectal cancer]. Zhonghua Wai Ke Za Zhi. 2005;43(9):564-8.

21. Szymanska E, Markuszewski MJ, Markuszewski M, Kaliszan R. Altered levels of nucleoside metabolite profiles in urogenital tract cancer measured by capillary electrophoresis. J Pharm Biomed Anal. 2010;53(5):1305-12.

22. Zhang T, Wu X, Ke C, Yin M, Li Z, Fan L, et al. Identification of potential biomarkers for ovarian cancer by urinary metabolomic profiling. J Proteome Res. 2013;12(1):505-12. 
23. Li H, Qin Q, Shi X, He J, Xu G. Modified metabolites mapping by liquid chromatography-high resolution mass spectrometry using full scan/all ion fragmentation/neutral loss acquisition. $J$ Chromatogr A. 2019;1583:80-7.

24. Lv J, Liu H, Wang Q, Tang Z, Hou L, Zhang B. Molecular cloning of a novel human gene encoding histone acetyltransferase-like protein involved in transcriptional activation of hTERT. Biochem Biophys Res Commun. 2003;311(2):506-13.

25. Tan TZ, Miow QH, Huang RY, Wong MK, Ye J, Lau JA, et al. Functional genomics identifies five distinct molecular subtypes with clinical relevance and pathways for growth control in epithelial ovarian cancer. EMBO Mol Med. 2013;5(7):1051-66.

26. Oh TI, Lee YM, Lim BO, Lim JH. Inhibition of NAT10 Suppresses Melanogenesis and Melanoma Growth by Attenuating Microphthalmia-Associated Transcription Factor (MITF) Expression. Int J Mol Sci. 2017;18(9).

27. Tschida BR, Temiz NA, Kuka TP, Lee LA, Riordan JD, Tierrablanca CA, et al. Sleeping Beauty Insertional Mutagenesis in Mice Identifies Drivers of Steatosis-Associated Hepatic Tumors. Cancer Res. 2017;77(23):6576-88.

28. Cao Y, Yao M, Wu Y, Ma N, Liu H, Zhang B. N-Acetyltransferase 10 Promotes Micronuclei Formation to Activate the Senescence-Associated Secretory Phenotype Machinery in Colorectal Cancer Cells. Transl Oncol. 2020;13(8):100783.

29. Duan J, Zhang Q, Hu X, Lu D, Yu W, Bai H. N(4)-acetylcytidine is required for sustained NLRP3 inflammasome activation via HMGB1 pathway in microglia. Cell Signal. 2019;58:44-52.

30. Doskocil J, Holy A. Inhibition of nucleoside-binding sites by nucleoside analogues in Escherichia coli. Nucleic Acids Res. 1974;1(3):491-502.

31. June CH. Adoptive T cell therapy for cancer in the clinic. J Clin Invest. 2007;117(6):1466-76.

32. Topalian SL, Drake CG, Pardoll DM. Immune checkpoint blockade: a common denominator approach to cancer therapy. Cancer Cell. 2015;27(4):450-61.

33. Yamamoto TN, Kishton RJ, Restifo NP. Developing neoantigen-targeted T cell-based treatments for solid tumors. Nat Med. 2019;25(10):1488-99.

34. Nikanjam M, Cohen PR, Kato S, Sicklick JK, Kurzrock R. Advanced basal cell cancer: concise review of molecular characteristics and novel targeted and immune therapeutics. Ann Oncol. 2018;29(11):2192-9.

35. Srinivas PR, Kramer BS, Srivastava S. Trends in biomarker research for cancer detection. Lancet Oncol. 2001;2(11):698-704.

36. Bindea G, Mlecnik B, Tosolini M, Kirilovsky A, Waldner M, Obenauf AC, et al. Spatiotemporal dynamics of intratumoral immune cells reveal the immune landscape in human cancer. Immunity. 2013;39(4):782-95.

37. Gajewski TF, Schreiber H, Fu YX. Innate and adaptive immune cells in the tumor microenvironment. Nat Immunol. 2013;14(10):1014-22. 
38. Tsai K, Jaguva Vasudevan AA, Martinez Campos C, Emery A, Swanstrom R, Cullen BR. Acetylation of Cytidine Residues Boosts HIV-1 Gene Expression by Increasing Viral RNA Stability. Cell Host Microbe. 2020;28(2):306-12 e6.

\section{Tables}

Due to technical limitations, table 1 is only available as a download in the Supplemental Files section.

\section{Figures}




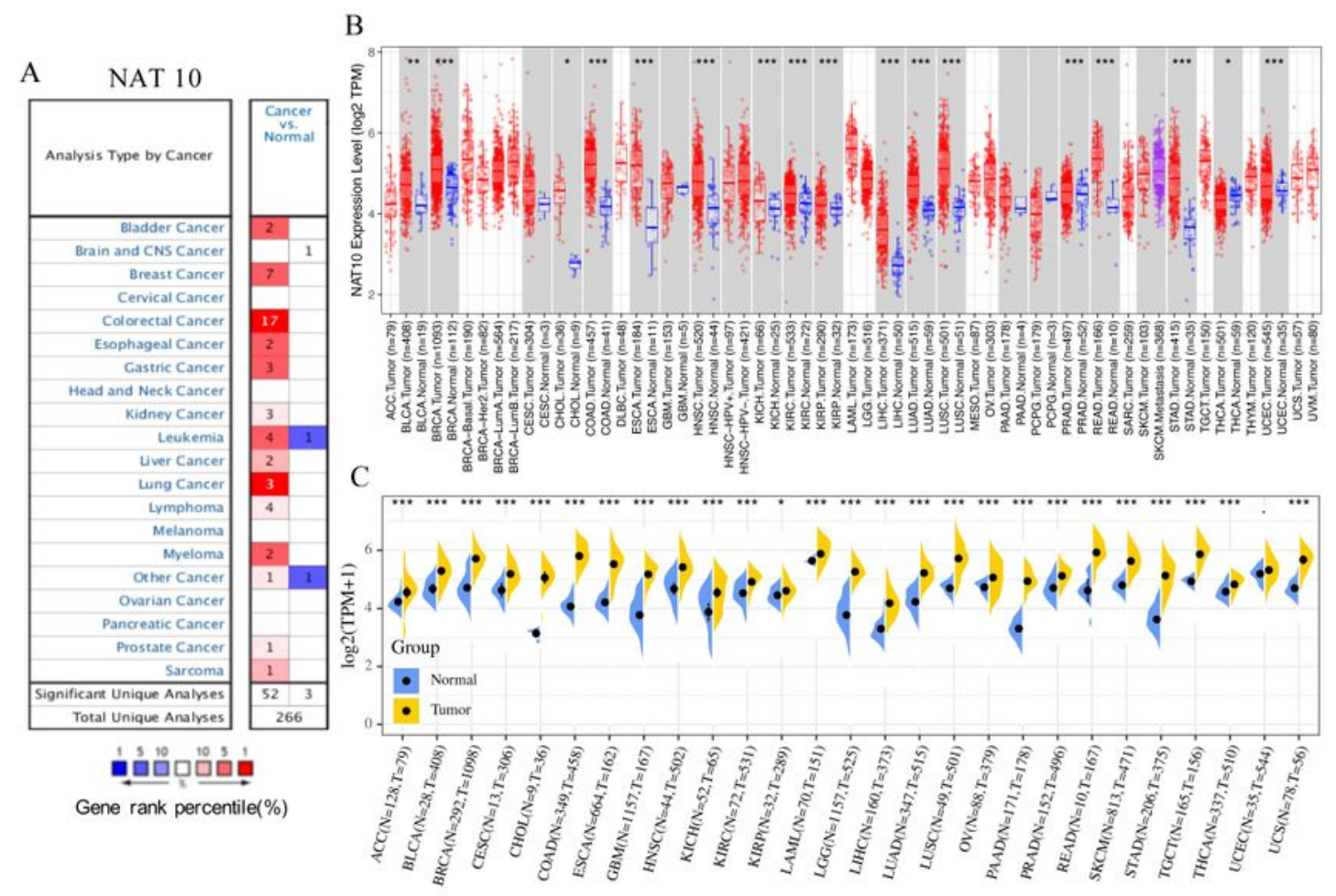

D Kruskal-Wallis test $\mathrm{p}=0$
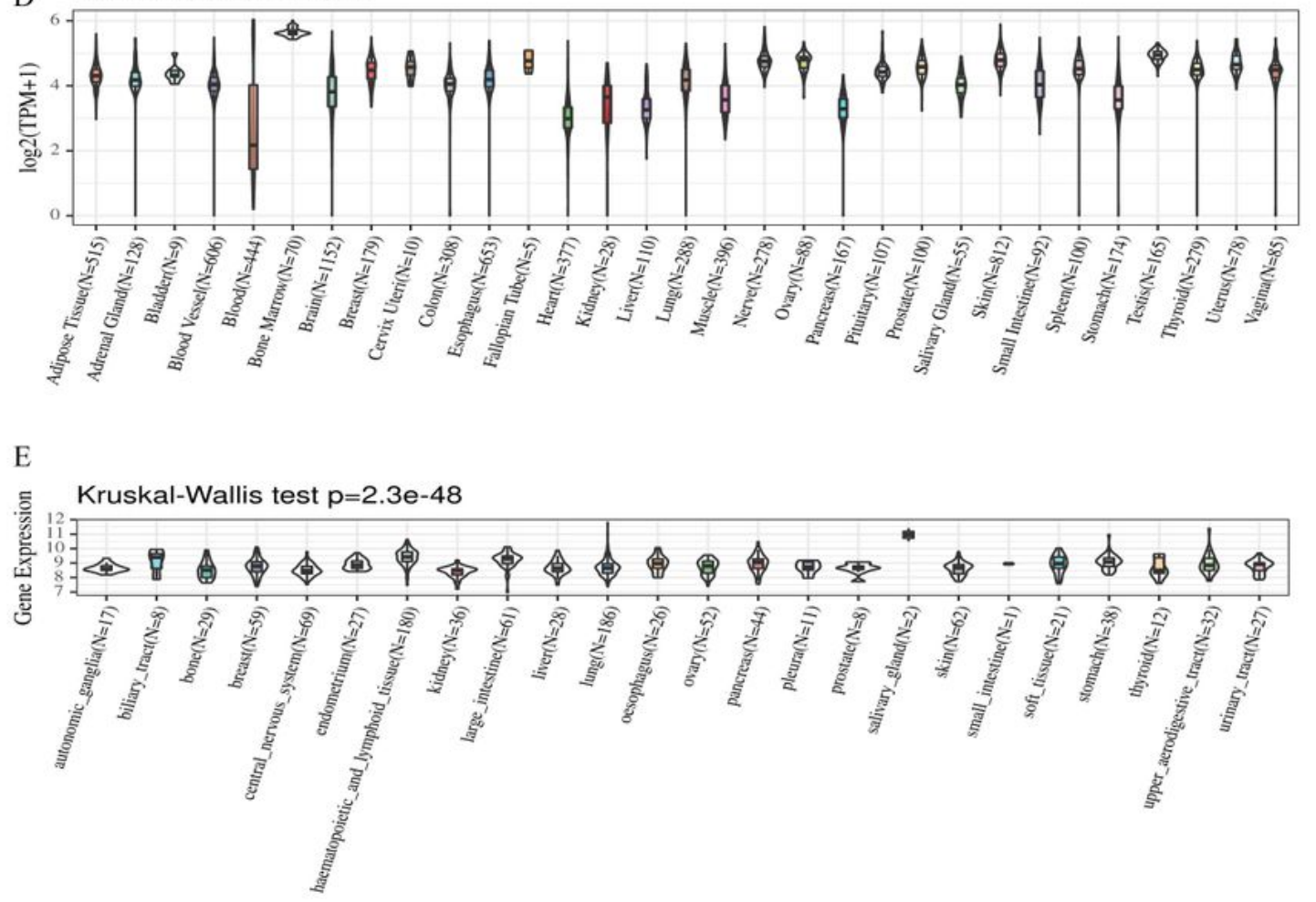

Figure 1

mRNA expression levels of NAT10 in different human cancers. (A) Increased or decreased expression of NAT10 in datasets for different cancer tissues, compared with normal tissues from the Oncomine database. The number in each cell is the size of the dataset. (B) Human NAT10 expression levels in different tumor types from TCGA database determined by TIMER. (C) Human NAT10 expression levels in normal, peri-tumor, and tumor samples, combining data from TCGA and GTEx. (D) Normal mRNA 
expression levels of NAT10 in different tissues from GTEx database. (E) NAT10 expression in various tumor cell lines from CCLE database. ( ${ }^{\star} P<0.05,{ }^{\star} \mathrm{P}<0.01$, $\left.{ }^{\star \star \star} \mathrm{P}<0.001\right)$.

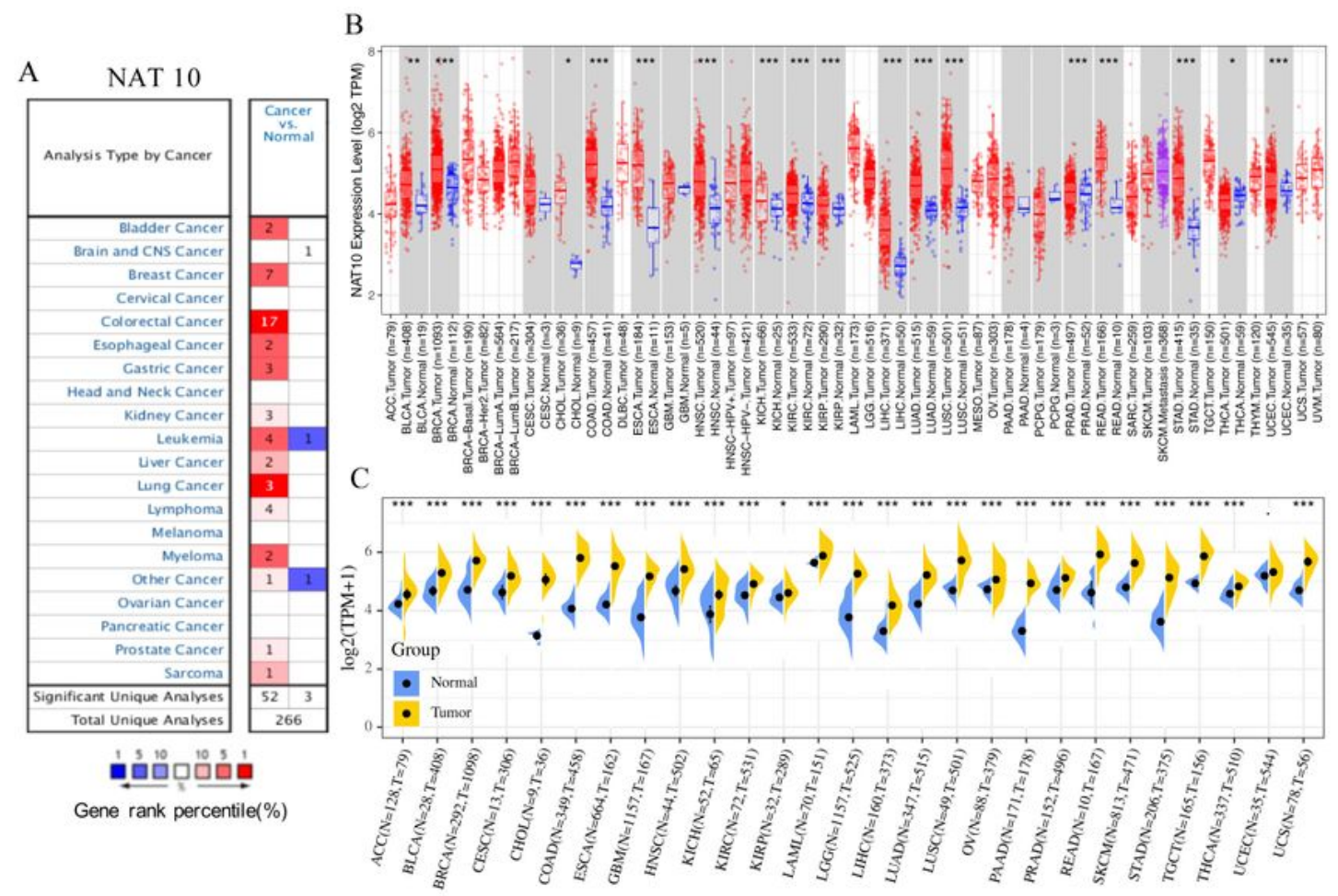

D Kruskal-Wallis test $\mathrm{p}=0$
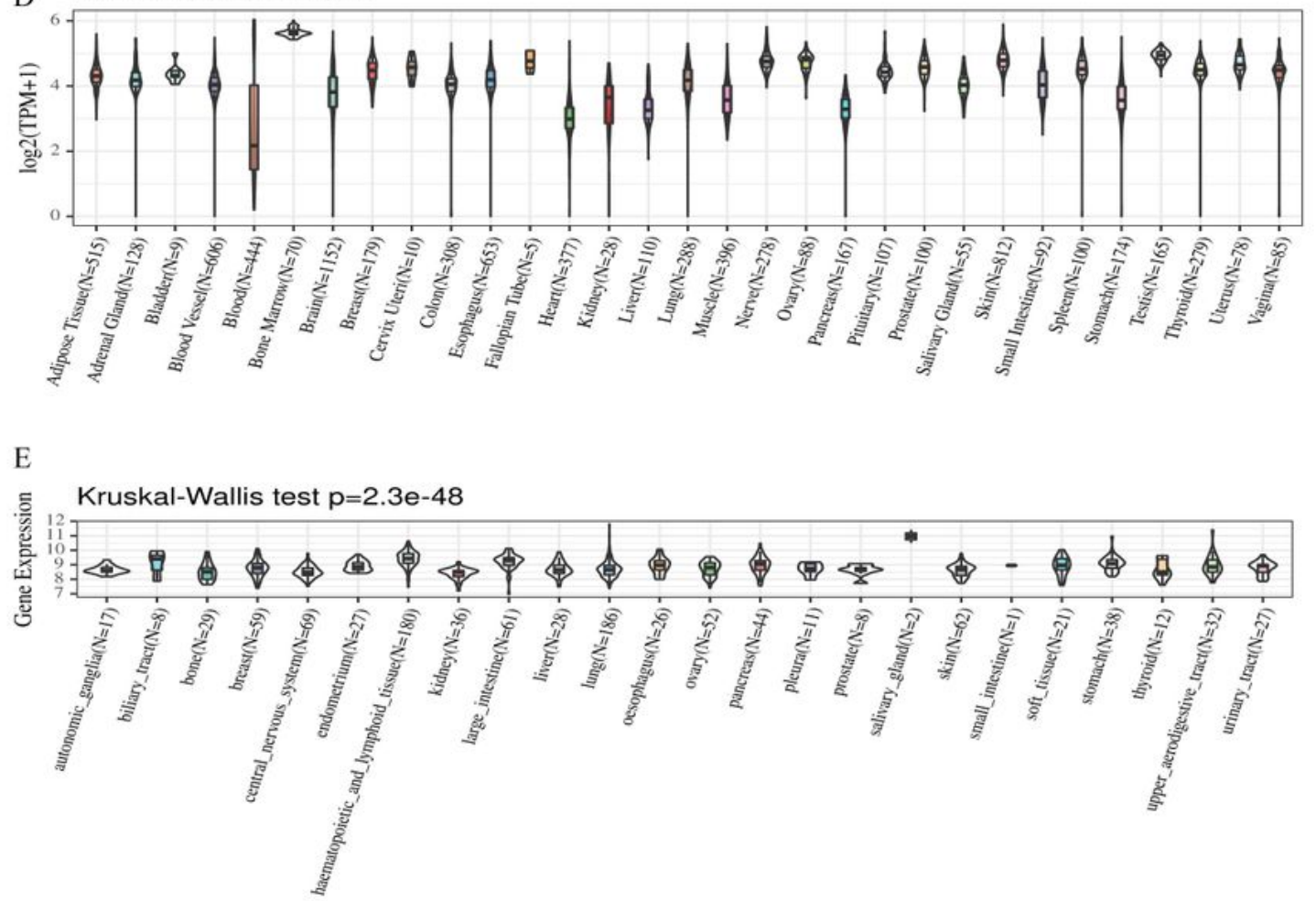

Figure 1

mRNA expression levels of NAT10 in different human cancers. (A) Increased or decreased expression of NAT10 in datasets for different cancer tissues, compared with normal tissues from the Oncomine database. The number in each cell is the size of the dataset. (B) Human NAT10 expression levels in 
different tumor types from TCGA database determined by TIMER. (C) Human NAT10 expression levels in normal, peri-tumor, and tumor samples, combining data from TCGA and GTEx. (D) Normal mRNA expression levels of NAT10 in different tissues from GTEx database. (E) NAT10 expression in various tumor cell lines from CCLE database. $\left({ }^{*} P<0.05,{ }^{*} \mathrm{P}<0.01,{ }^{*} * * \mathrm{P}<0.001\right)$.

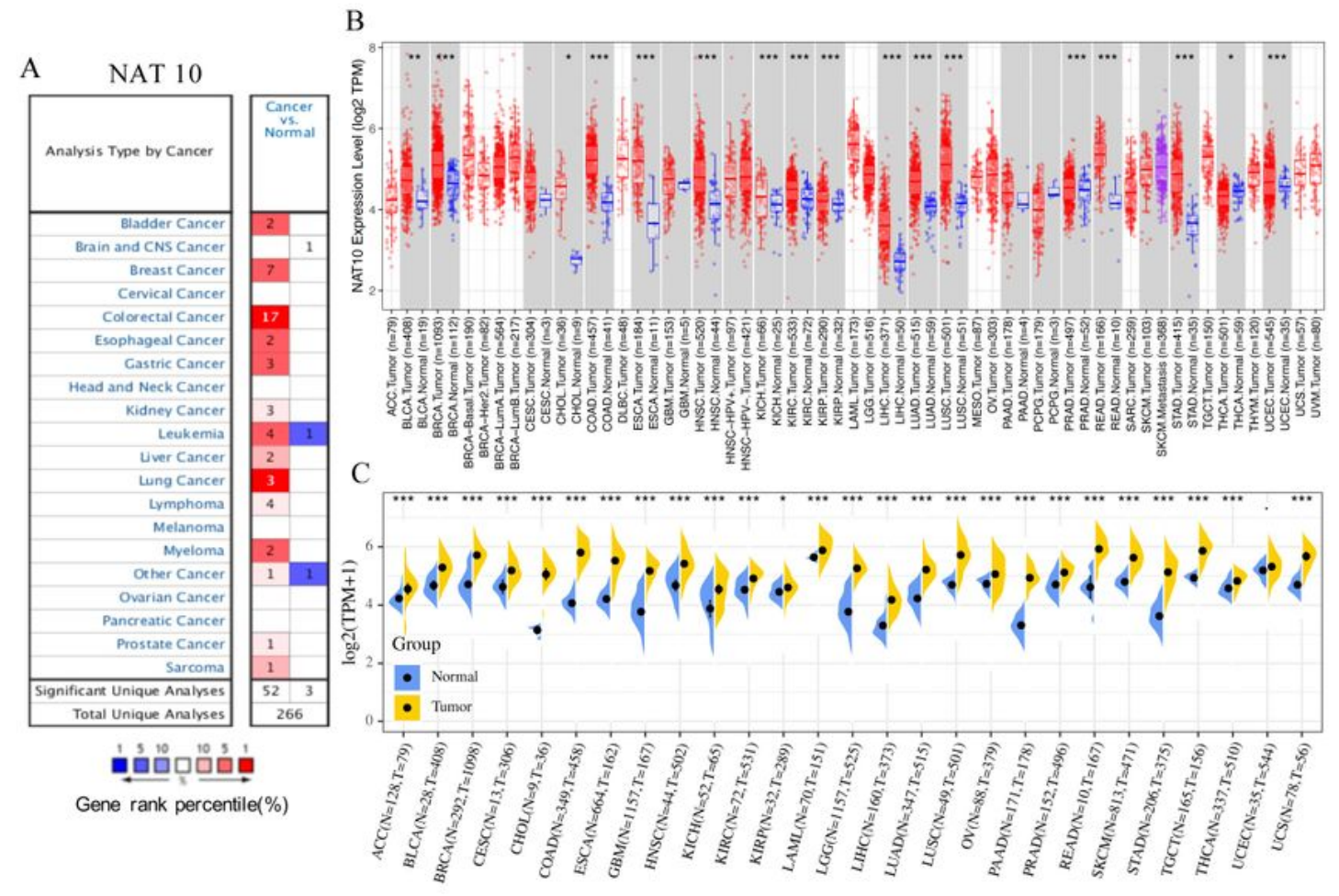

D Kruskal-Wallis test $\mathrm{p}=0$
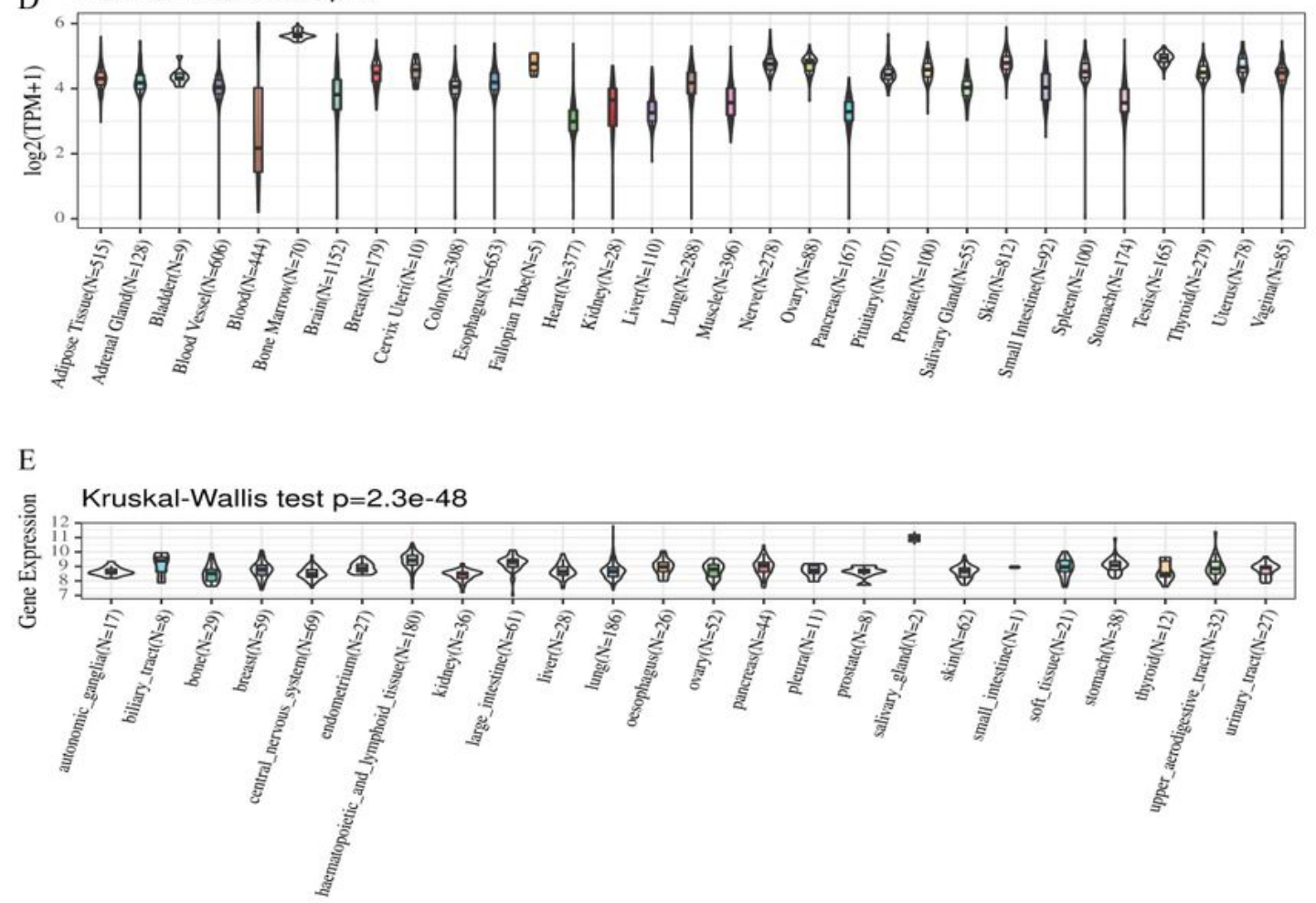

Figure 1 
mRNA expression levels of NAT10 in different human cancers. (A) Increased or decreased expression of NAT10 in datasets for different cancer tissues, compared with normal tissues from the Oncomine database. The number in each cell is the size of the dataset. (B) Human NAT10 expression levels in different tumor types from TCGA database determined by TIMER. (C) Human NAT10 expression levels in normal, peri-tumor, and tumor samples, combining data from TCGA and GTEx. (D) Normal mRNA expression levels of NAT10 in different tissues from GTEx database. (E) NAT10 expression in various tumor cell lines from CCLE database. $\left({ }^{*} P<0.05,{ }^{*} \mathrm{P}<0.01\right.$, $\left.{ }^{* *} \mathrm{P}<0.001\right)$.

A

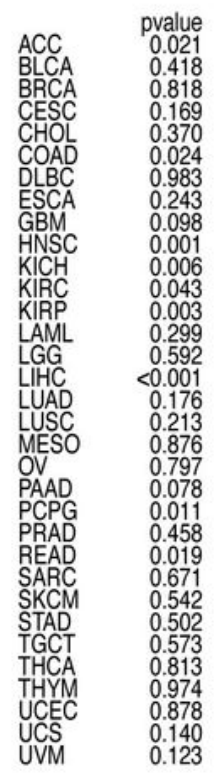

$\mathrm{C}$

$\begin{array}{lrr} & \text { pvalue } & \text { Hazard ratio } \\ \text { ACC } & 0.006 & 10.020(1.949-51.516) \\ \text { BLCA } & 0.700 & 0.866(0.417-1.798) \\ \text { BRCA } & 0.654 & 1.092(0.744-1.602) \\ \text { CESC } & 0.536 & 1.343(0.528-3.417) \\ \text { CHOL } & 0.711 & 1.165(0.518-2.622) \\ \text { COAD } & 0.009 & 2.463(1.253-4.843) \\ \text { DLBC } & 0.454 & 0.194(0.003-14.113) \\ \text { ESCA } & 0.634 & 1.230(0.524-2.886) \\ \text { HNSC } & 0.110 & 1.833(0.872-3.852) \\ \text { KICH } & 0.315 & 0.247(0.016-3.788) \\ \text { KIRC } & 0.362 & 0.537(0.141-2.043) \\ \text { KIRP } & <0.001 & 11.302(2.982-42.836) \\ \text { LGG } & 0.159 & 2.583(0.690-9.676) \\ \text { LIHC } & 0.005 & 1.546(1.138-2.100) \\ \text { LUAD } & 0.125 & 1.382(0.914-2.091) \\ \text { LUSC } & 0.945 & 1.014(0.688-1.493) \\ \text { MESO } & 0.646 & 0.338(0.003-34.480) \\ \text { OV } & 0.003 & 0.666(0.508-0.875) \\ \text { PAAD } & 0.223 & 1.884(0.681-5.214) \\ \text { PCPG } & 0.093 & 9.736(0.683-138.695) \\ \text { PRAD } & 0.171 & 2.426(0.682-8.620) \\ \text { READ } & 0.681 & 0.711(0.139-3.623) \\ \text { SARC } & 0.754 & 1.070(0.700-1.635) \\ \text { STAD } & 0.120 & 0.657(0.387-1.116) \\ \text { TGCT } & 0.523 & 1.325(0.558-3.146) \\ \text { THCA } & 0.718 & 1.398(0.227-8.626) \\ \text { UCEC } & 0.829 & 1.070(0.576-1.988) \\ \text { UCS } & 0.473 & 2.010(0.298-13.557)\end{array}$

OS

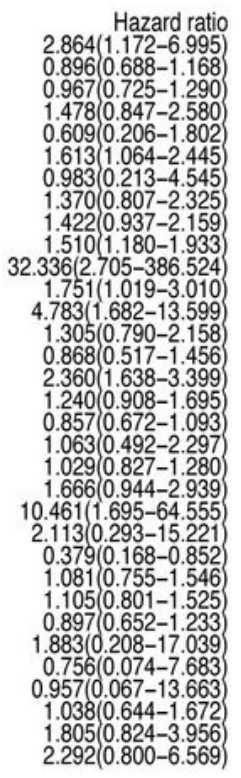

DFS

$2.010(0.298-13.557)$

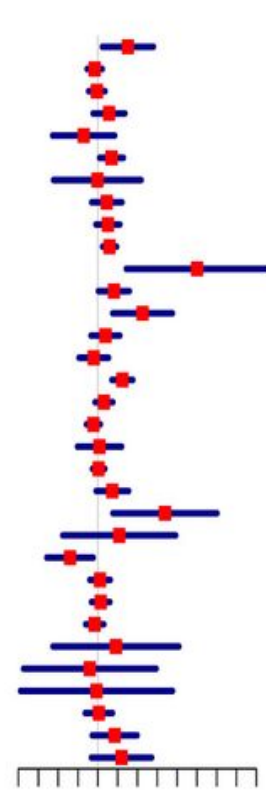

$0.062 \quad 1 e+002 e+013 e+02$ Hazard ratio
B

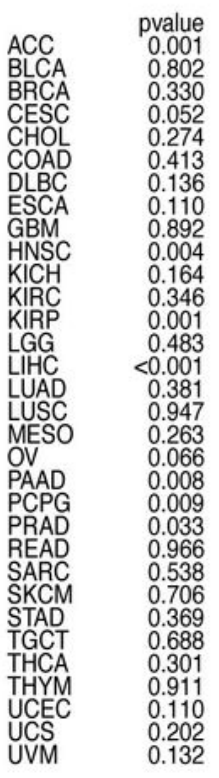

D

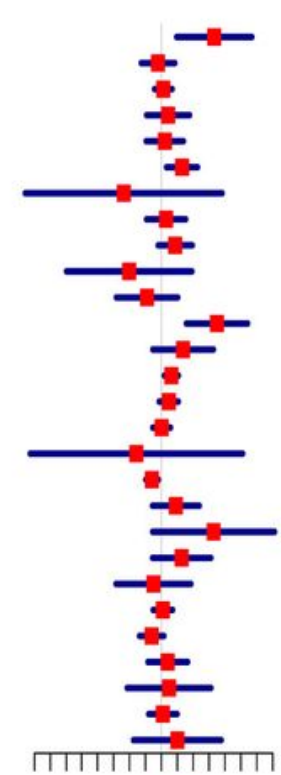

$\begin{array}{lllll}0.004 & 0.125 & 4 \mathrm{e}+00 & 1 \mathrm{e}+02\end{array}$ Hazard ratio

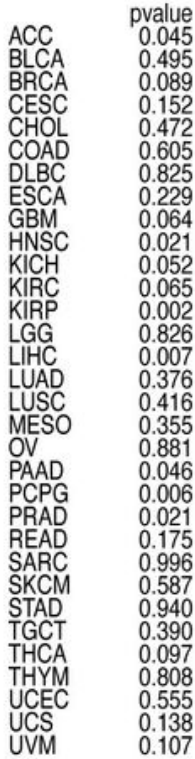

\section{PFS}
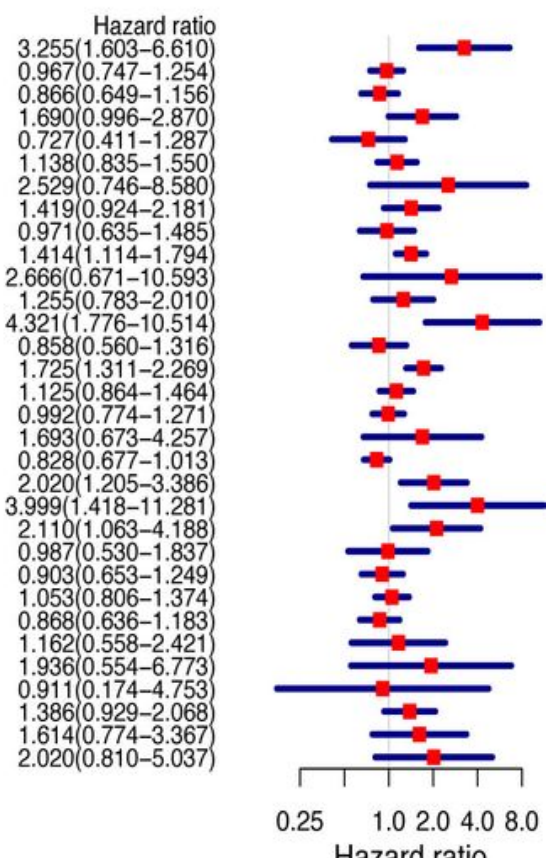

DSS
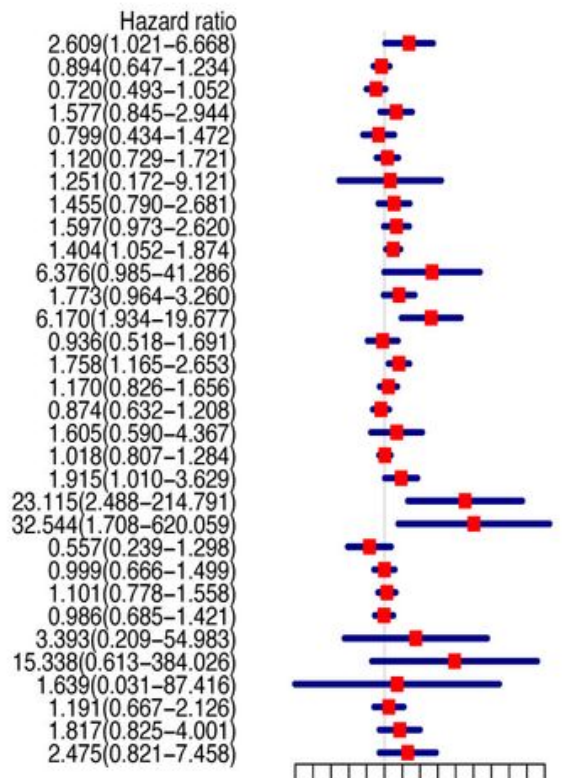

$0.0310 .5008 e+001 e+02$ Hazard ratio 
Figure 2

Association between NAT10 expression levels and patient prognosis based on multiple tumors from TCGA database. (A) Relationship of NAT10 expression with OS. (B) Relationship of NAT10 expression with progression-free interval (PFI). (C) Relationship of NAT10 expression with disease-free interval (DFI). (D) Relationship of NAT10 expression with DSS. Cox regression analysis; $\mathrm{P}<0.05$ was considered significant.

A

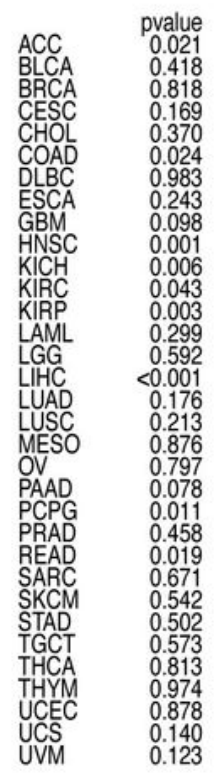

$\mathrm{C}$

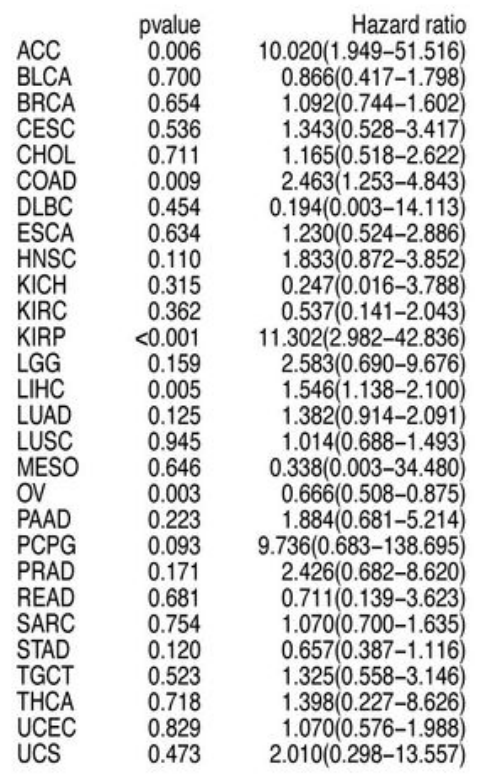

OS

DFS

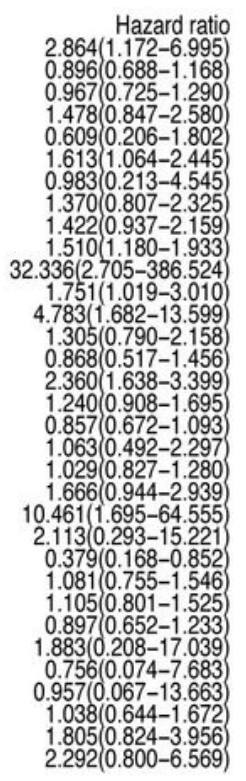

\section{$0.0621 e+002 e+013 e+02$ Hazard ratio}
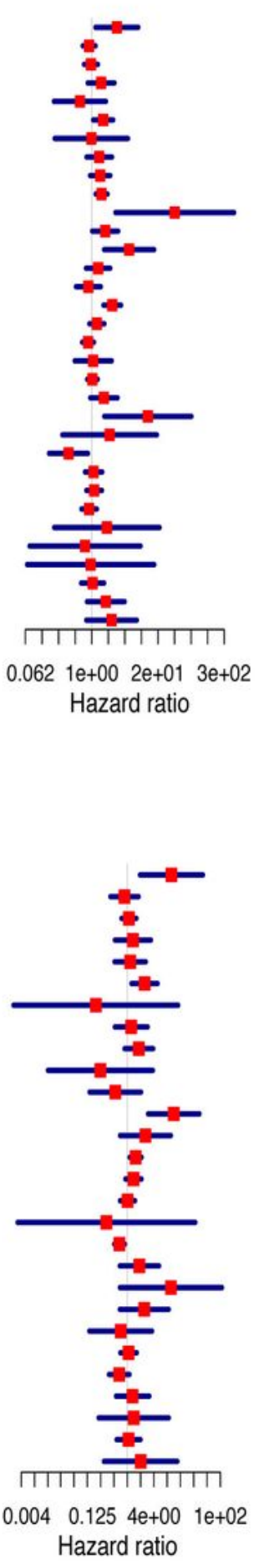

B

$\begin{array}{lr} & \text { pvalue } \\ \text { ACC } & 0.001 \\ \text { BLCA } & 0.802 \\ \text { BRCA } & 0.330 \\ \text { CESC } & 0.052 \\ \text { CHOL } & 0.274 \\ \text { COAD } & 0.413 \\ \text { DLBC } & 0.136 \\ \text { ESCA } & 0.110 \\ \text { GBM } & 0.892 \\ \text { HNSC } & 0.004 \\ \text { KICH } & 0.164 \\ \text { KIRC } & 0.346 \\ \text { KIRP } & 0.001 \\ \text { LGG } & 0.483 \\ \text { LIHC } & <0.001 \\ \text { LUAD } & 0.381 \\ \text { LUSC } & 0.947 \\ \text { MESO } & 0.263 \\ \text { OV } & 0.066 \\ \text { PAAD } & 0.008 \\ \text { PCPG } & 0.009 \\ \text { PRAD } & 0.033 \\ \text { READ } & 0.966 \\ \text { SARC } & 0.538 \\ \text { SKCM } & 0.706 \\ \text { STAD } & 0.369 \\ \text { TGCT } & 0.688 \\ \text { THCA } & 0.301 \\ \text { THYM } & 0.911 \\ \text { UCEC } & 0.110 \\ \text { UCS } & 0.202 \\ \text { UVM } & 0.132 \\ \end{array}$

D

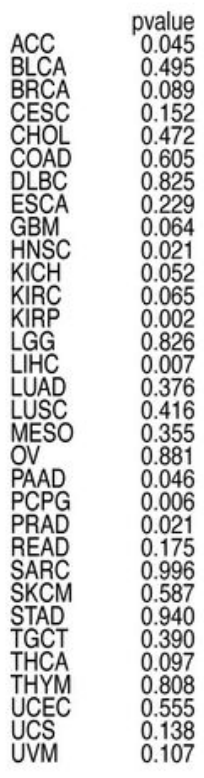

\section{PFS}

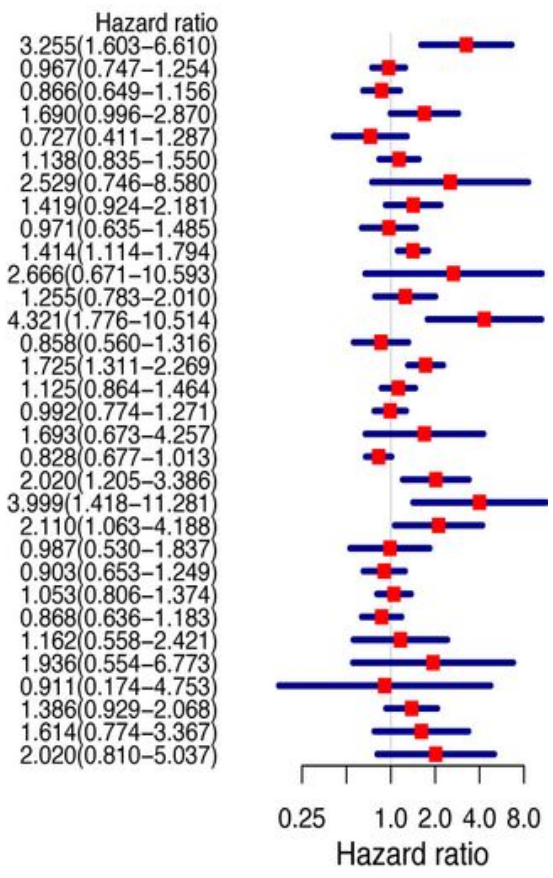

DSS
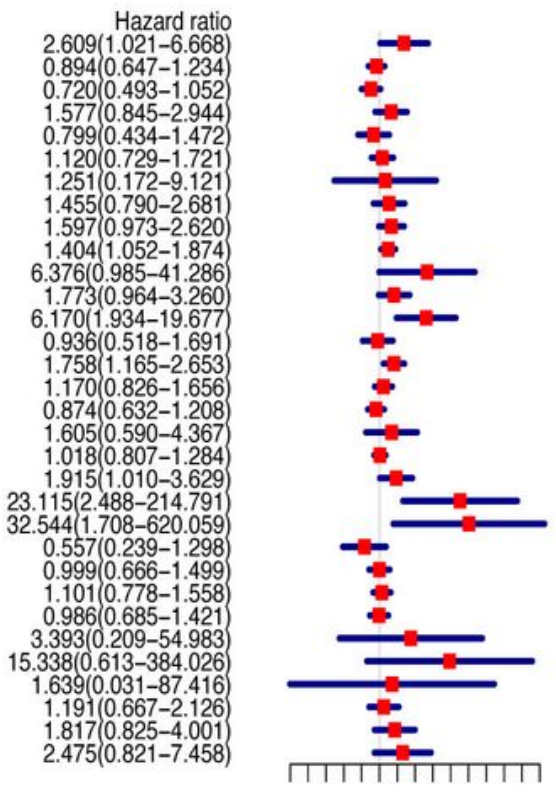

$0.0310 .5008 e+001 e+02$ Hazard ratio 
Figure 2

Association between NAT10 expression levels and patient prognosis based on multiple tumors from TCGA database. (A) Relationship of NAT10 expression with OS. (B) Relationship of NAT10 expression with progression-free interval (PFI). (C) Relationship of NAT10 expression with disease-free interval (DFI). (D) Relationship of NAT10 expression with DSS. Cox regression analysis; $\mathrm{P}<0.05$ was considered significant.

A

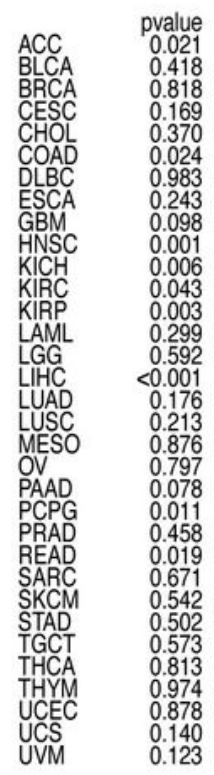

$\mathrm{C}$

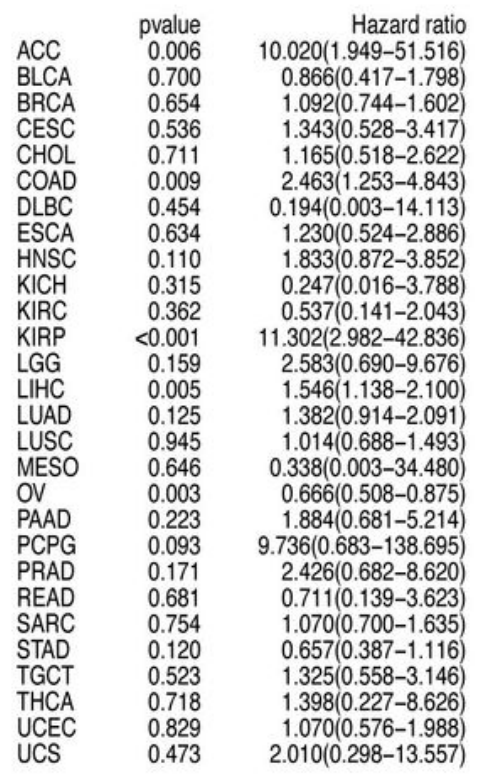

OS

DFS

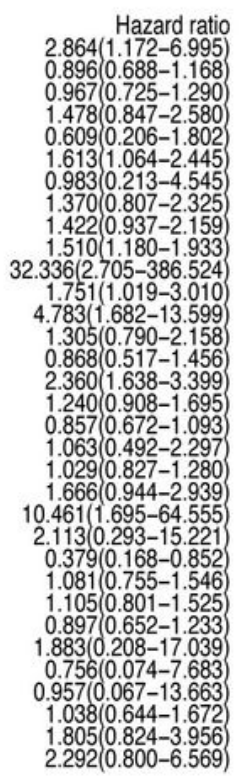

\section{$0.0621 e+002 e+013 e+02$ Hazard ratio}
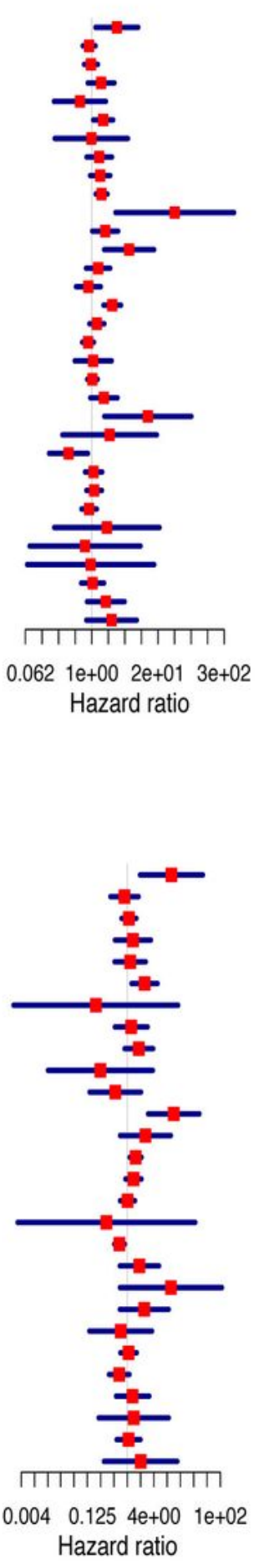

B

$\begin{array}{lr} & \text { pvalue } \\ \text { ACC } & 0.001 \\ \text { BLCA } & 0.802 \\ \text { BRCA } & 0.330 \\ \text { CESC } & 0.052 \\ \text { CHOL } & 0.274 \\ \text { COAD } & 0.413 \\ \text { DLBC } & 0.136 \\ \text { ESCA } & 0.110 \\ \text { GBM } & 0.892 \\ \text { HNSC } & 0.004 \\ \text { KICH } & 0.164 \\ \text { KIRC } & 0.346 \\ \text { KIRP } & 0.001 \\ \text { LGG } & 0.483 \\ \text { LIHC } & <0.001 \\ \text { LUAD } & 0.381 \\ \text { LUSC } & 0.947 \\ \text { MESO } & 0.263 \\ \text { OV } & 0.066 \\ \text { PAAD } & 0.008 \\ \text { PCPG } & 0.009 \\ \text { PRAD } & 0.033 \\ \text { READ } & 0.966 \\ \text { SARC } & 0.538 \\ \text { SKCM } & 0.706 \\ \text { STAD } & 0.369 \\ \text { TGCT } & 0.688 \\ \text { THCA } & 0.301 \\ \text { THYM } & 0.911 \\ \text { UCEC } & 0.110 \\ \text { UCS } & 0.202 \\ \text { UVM } & 0.132 \\ \end{array}$

D

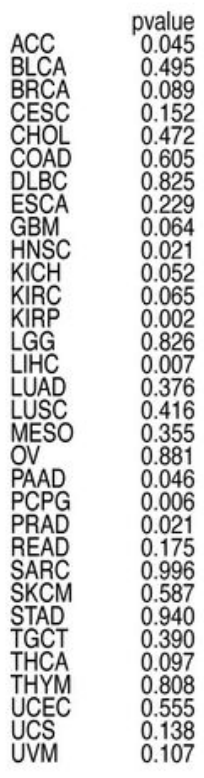

\section{PFS}

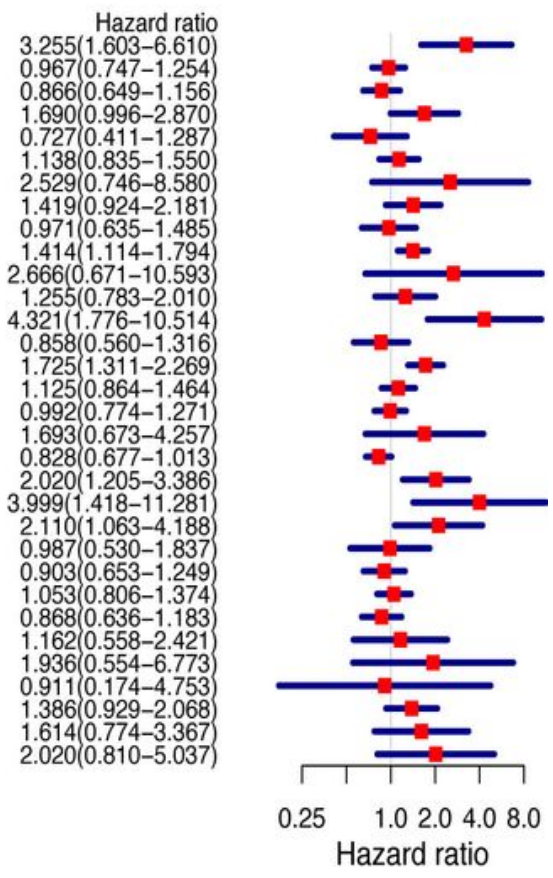

DSS
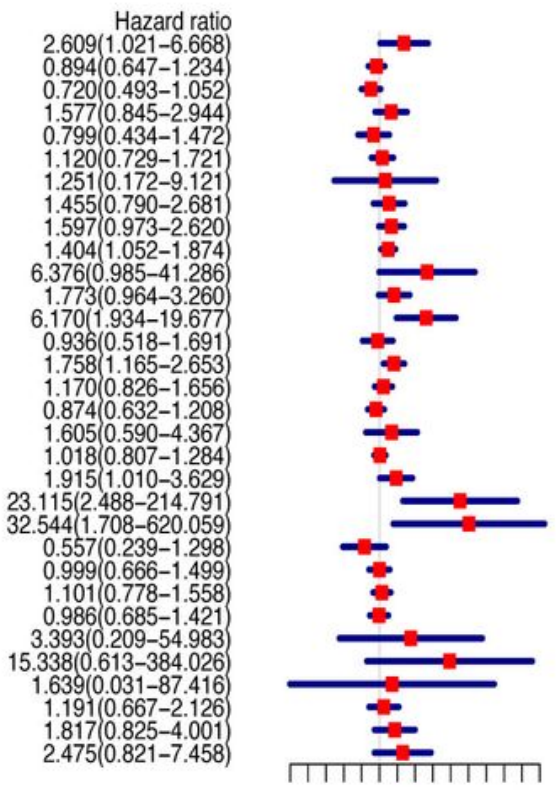

$0.0310 .5008 e+001 e+02$ Hazard ratio 


\section{Figure 2}

Association between NAT10 expression levels and patient prognosis based on multiple tumors from TCGA database. (A) Relationship of NAT10 expression with OS. (B) Relationship of NAT10 expression with progression-free interval (PFI). (C) Relationship of NAT10 expression with disease-free interval (DFI). (D) Relationship of NAT10 expression with DSS. Cox regression analysis; $\mathrm{P}<0.05$ was considered significant.

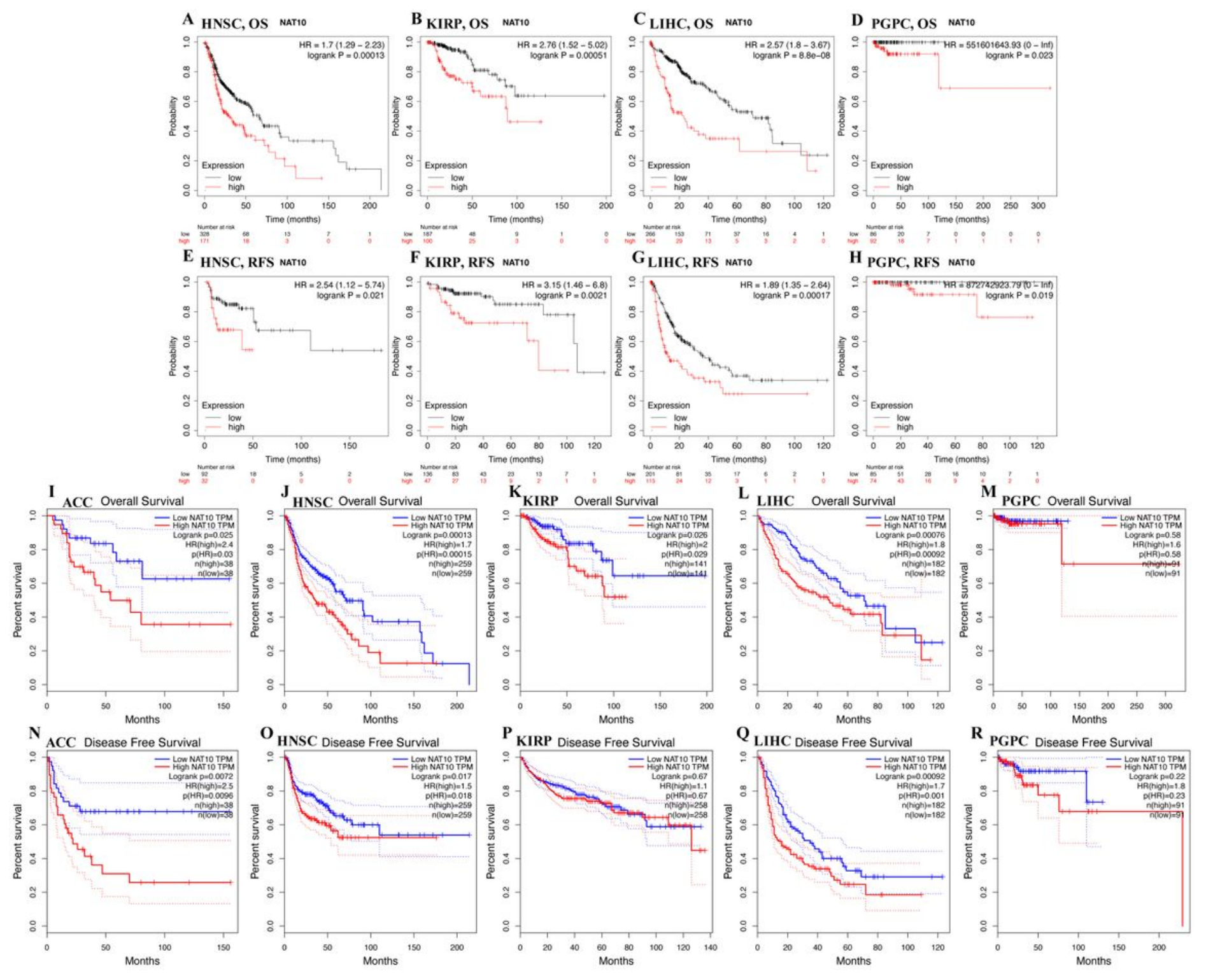

\section{Figure 3}

Kaplan-Meier survival curves comparing NAT10 expression in ACC, HNSC, KIRP, LIHC, and PCPG in GEPIA and Kaplan-Meier Plotter. (A-D) Differences in OS among groups in HNSC (A), KIRP (B), LIHC (C), and PCPG (D) in Kaplan-Meier Plotter. (E-H) Differences in RFS among groups in HNSC (E), KIRP (F), LIHC (G), and PCPG (H) in Kaplan-Meier Plotter. (I-M) Differences in OS among groups in ACC (I), HNSC (J), KIRP $(\mathrm{K})$, LIHC (L), and PCPG (M) in GEPIA. (N-R) Differences in DFS among groups in ACC (N), HNSC (O), KIRP $(P), \operatorname{LIHC}(\mathrm{Q})$, and PCPG (R) in GEPIA. 


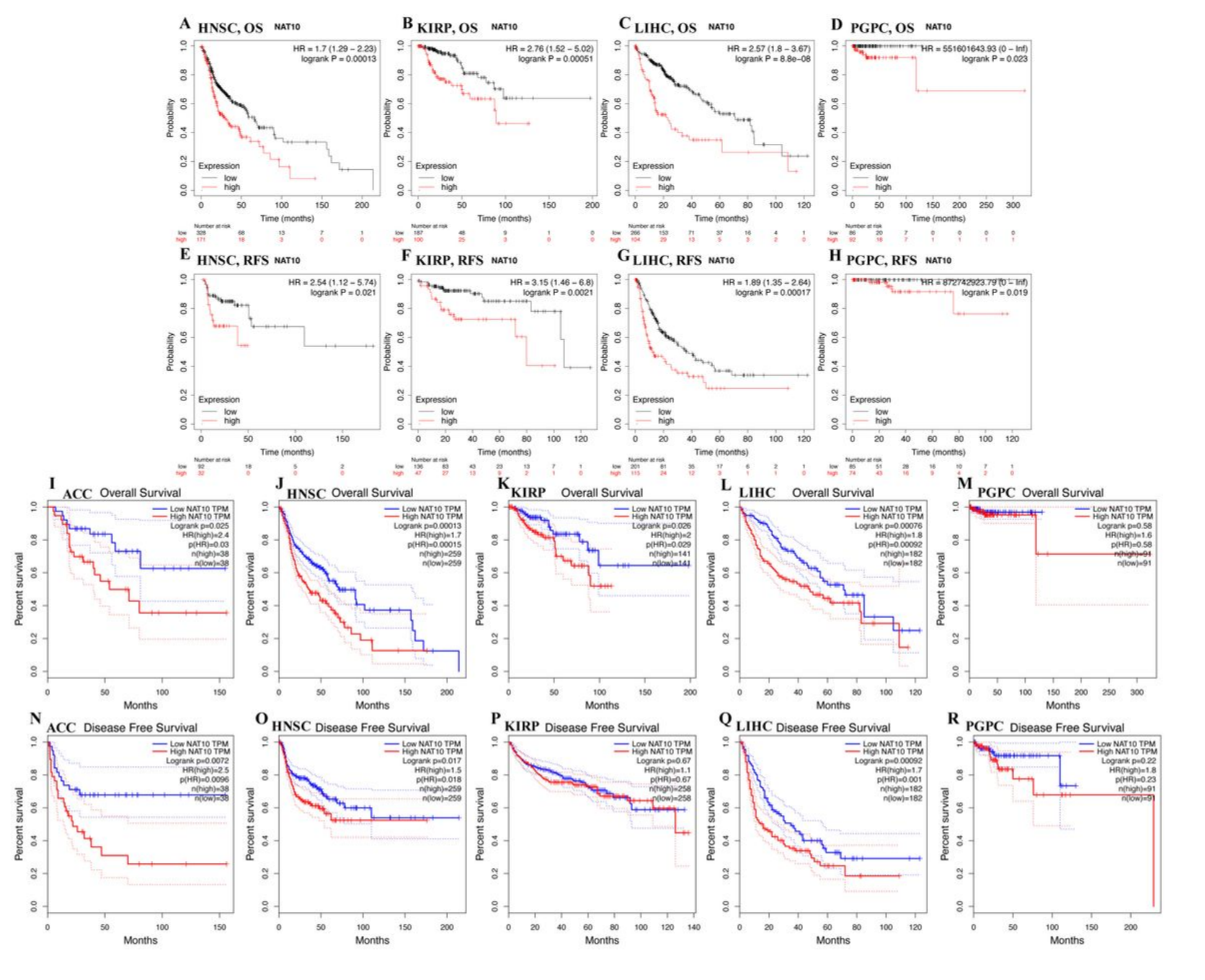

Figure 3

Kaplan-Meier survival curves comparing NAT10 expression in ACC, HNSC, KIRP, LIHC, and PCPG in GEPIA and Kaplan-Meier Plotter. (A-D) Differences in OS among groups in HNSC (A), KIRP (B), LIHC (C), and PCPG (D) in Kaplan-Meier Plotter. (E-H) Differences in RFS among groups in HNSC (E), KIRP (F), LIHC (G), and PCPG (H) in Kaplan-Meier Plotter. (I-M) Differences in OS among groups in ACC (I), HNSC (J), KIRP $(\mathrm{K})$, LIHC (L), and PCPG (M) in GEPIA. (N-R) Differences in DFS among groups in ACC (N), HNSC (O), KIRP $(P), \operatorname{LIHC}(\mathrm{Q})$, and PCPG (R) in GEPIA. 


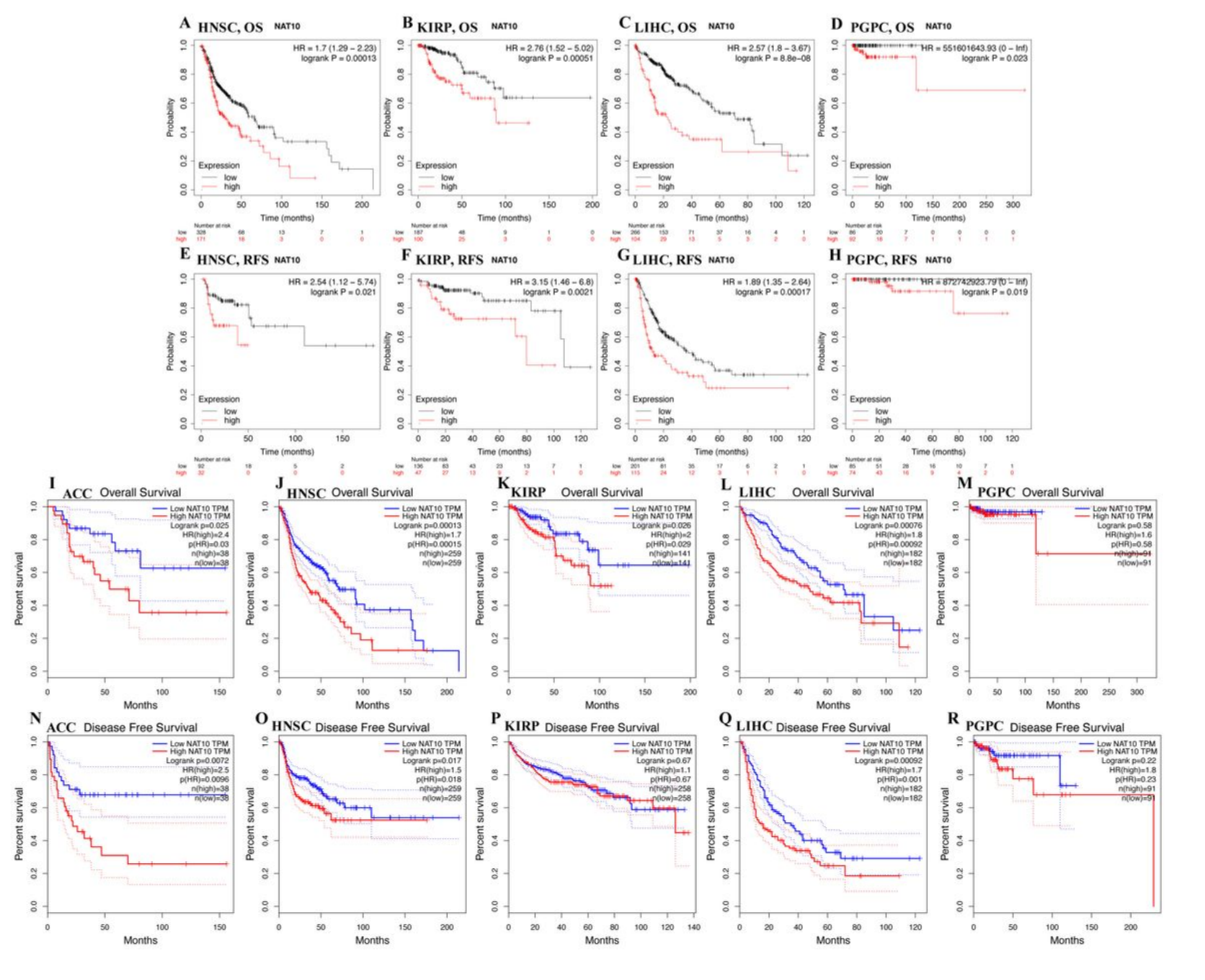

Figure 3

Kaplan-Meier survival curves comparing NAT10 expression in ACC, HNSC, KIRP, LIHC, and PCPG in GEPIA and Kaplan-Meier Plotter. (A-D) Differences in OS among groups in HNSC (A), KIRP (B), LIHC (C), and PCPG (D) in Kaplan-Meier Plotter. (E-H) Differences in RFS among groups in HNSC (E), KIRP (F), LIHC (G), and PCPG (H) in Kaplan-Meier Plotter. (I-M) Differences in OS among groups in ACC (I), HNSC (J), KIRP $(\mathrm{K})$, LIHC (L), and PCPG (M) in GEPIA. (N-R) Differences in DFS among groups in ACC (N), HNSC (O), KIRP $(P)$, LIHC (Q), and PCPG (R) in GEPIA. 
A

Cancer: ACC

Stage

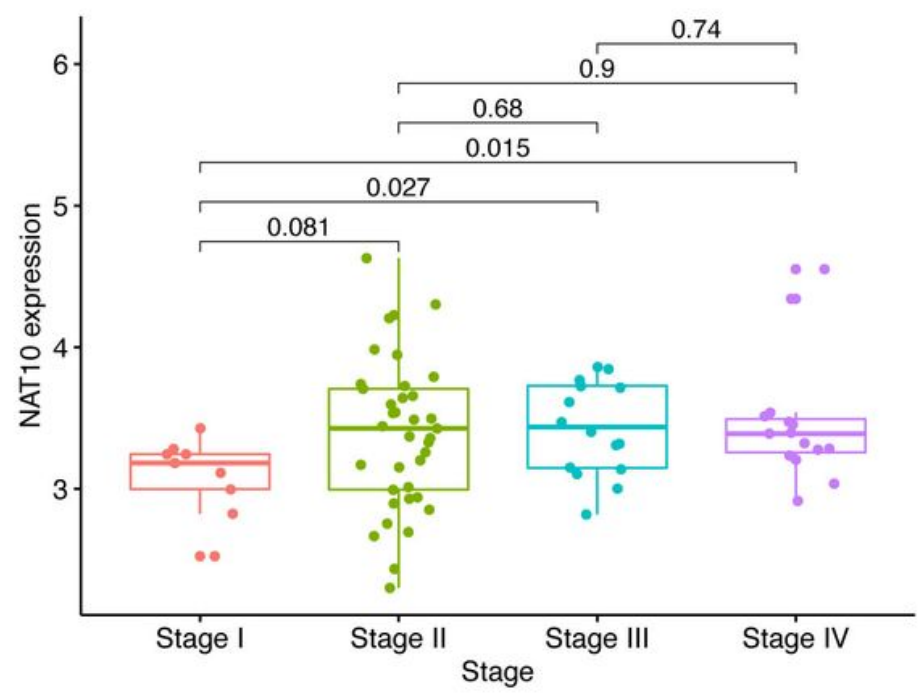

Cancer: KIRP

Stage Stage I 审 Stage II

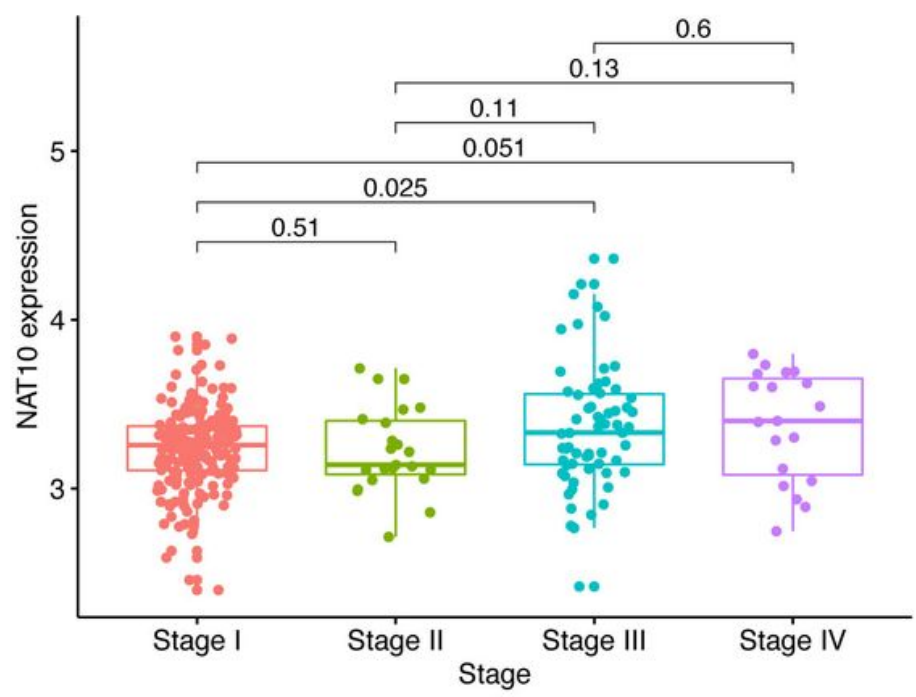

B Cancer: HNSC

Stage 审 Stage I 审 Stage II 审 Stage III 宊 Stage IV

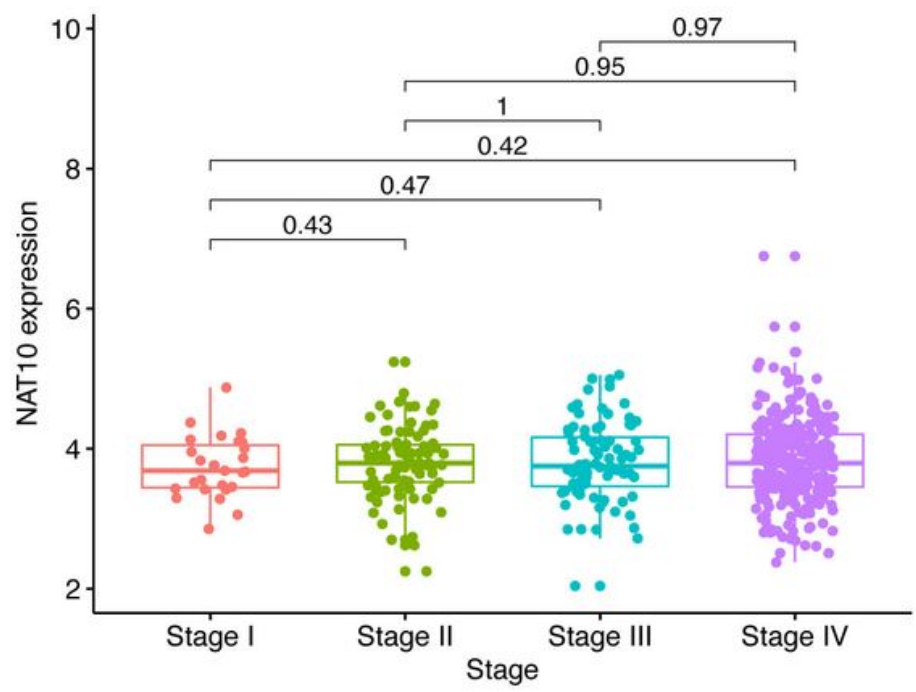

$\mathbf{D}_{\text {Cancer: LIHC }}$

Stage

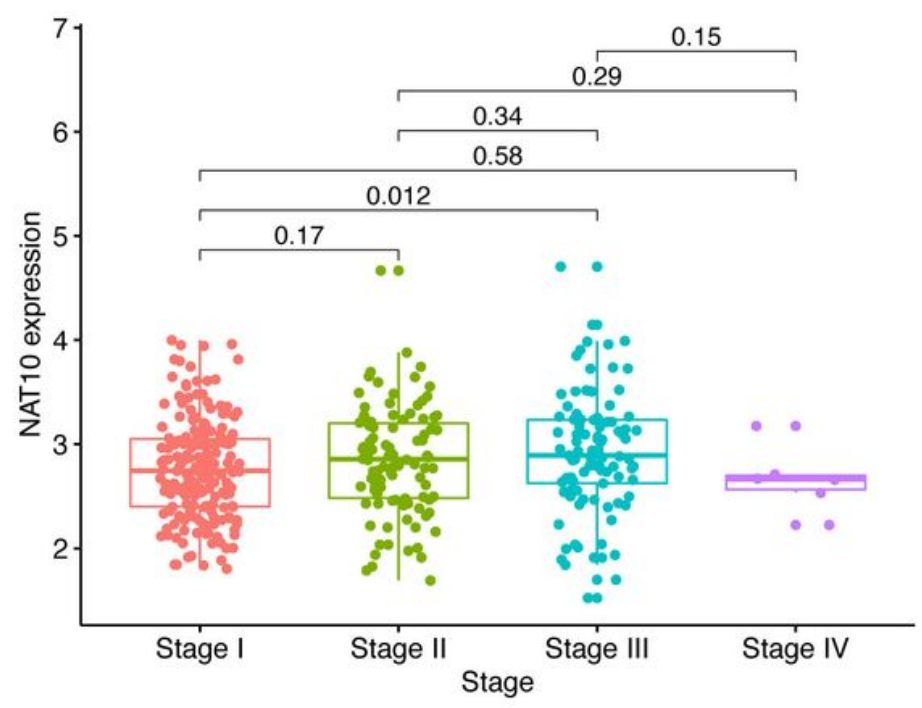

Figure 4

Correlation of NAT10 expression levels with stage in ACC, HNSC, KIRP, and LIHC. Relationships of NAT10 expression with stage in ACC (A), HNSC (B), KIRP (C), and LIHC (D). 
A

Cancer: ACC

Stage

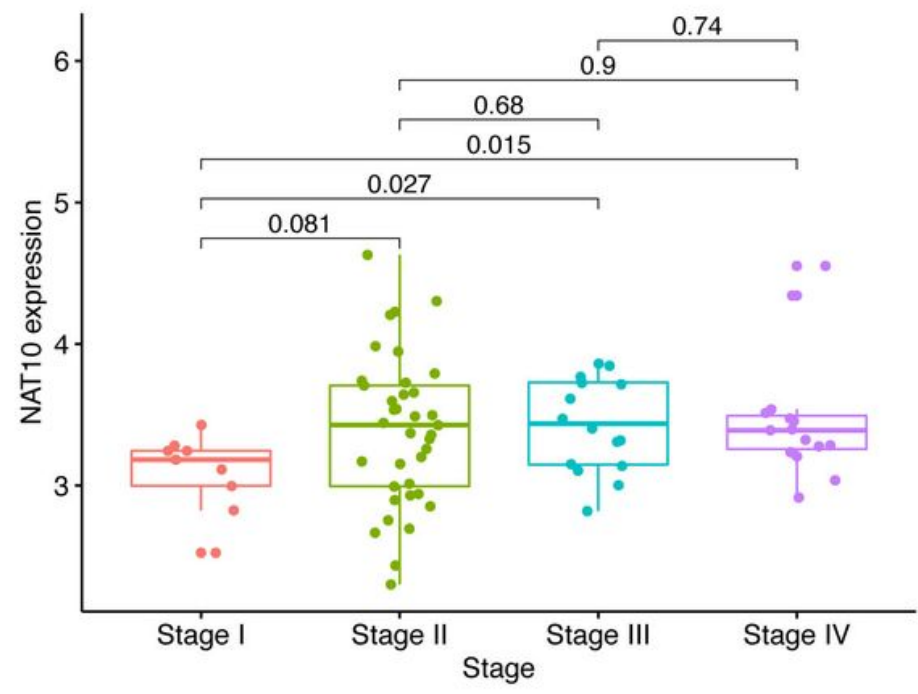

$C_{\text {Cancer: KIRP }}$

Stage Stage I 审 Stage II

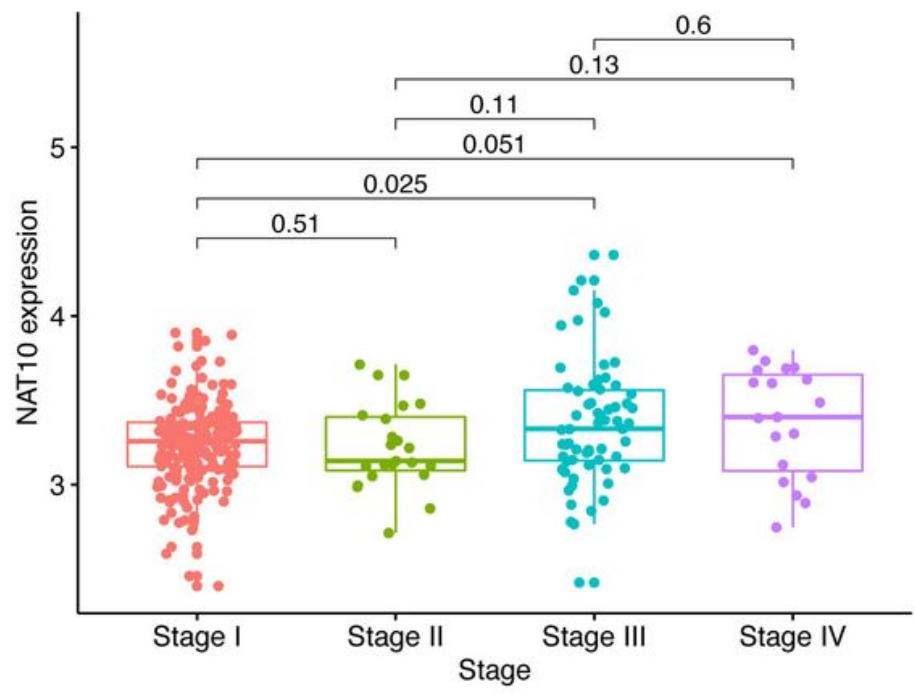

B Cancer: HNSC

Stage Stage I

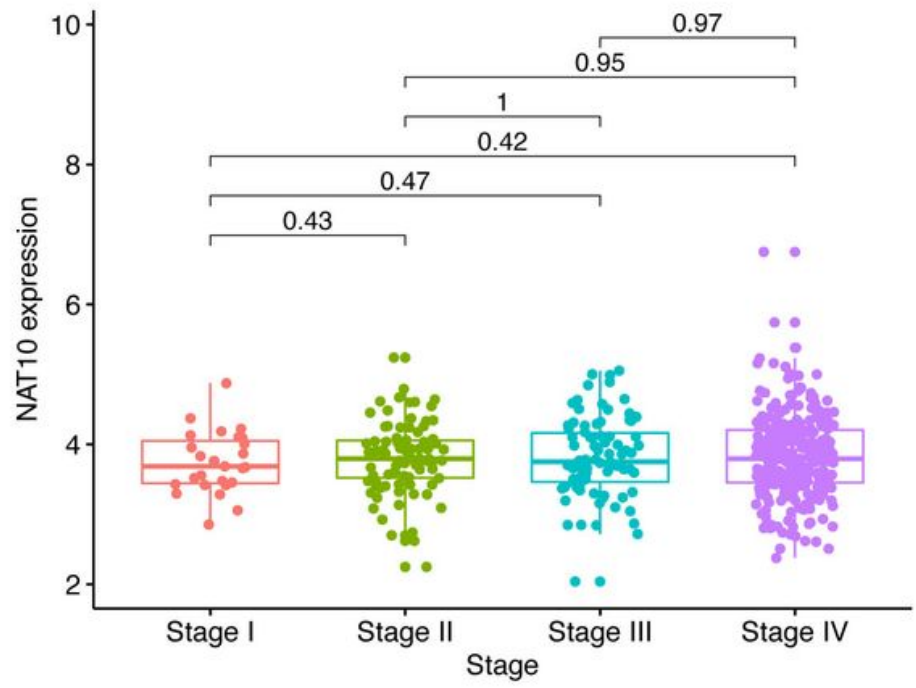

$D_{\text {Cancer: LIHC }}$

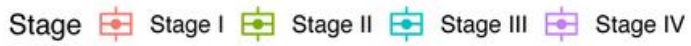

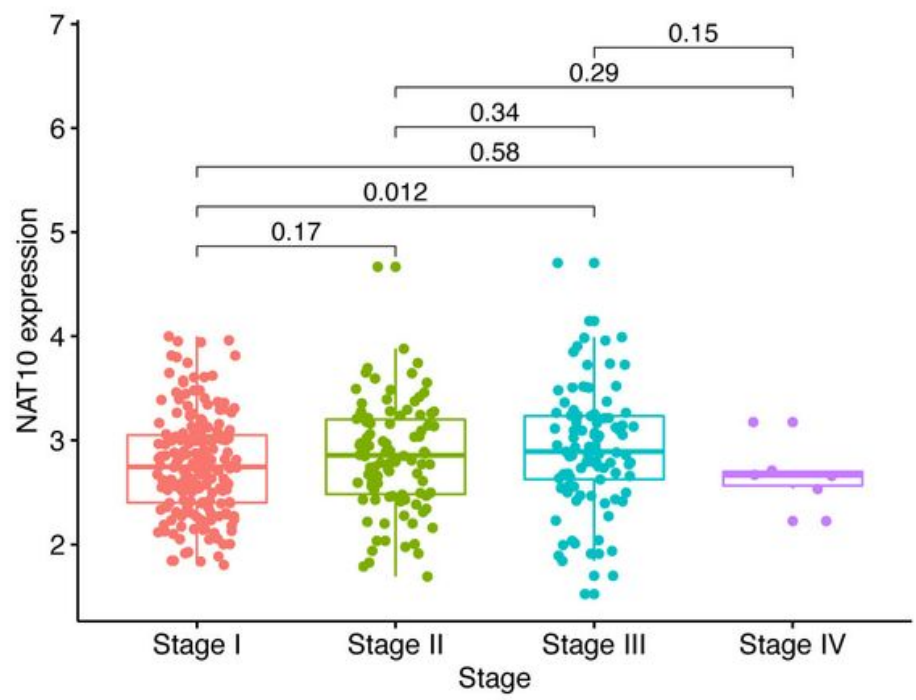

Figure 4

Correlation of NAT10 expression levels with stage in ACC, HNSC, KIRP, and LIHC. Relationships of NAT10 expression with stage in ACC (A), HNSC (B), KIRP (C), and LIHC (D). 
A

Cancer: ACC

Stage

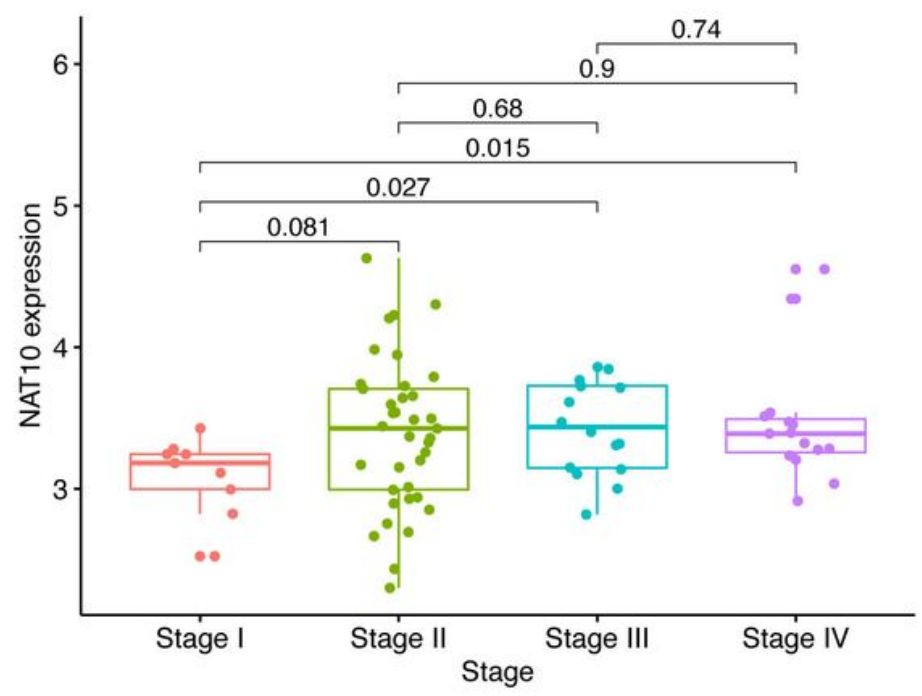

Cancer: KIRP

Stage Stage I 审 Stage II

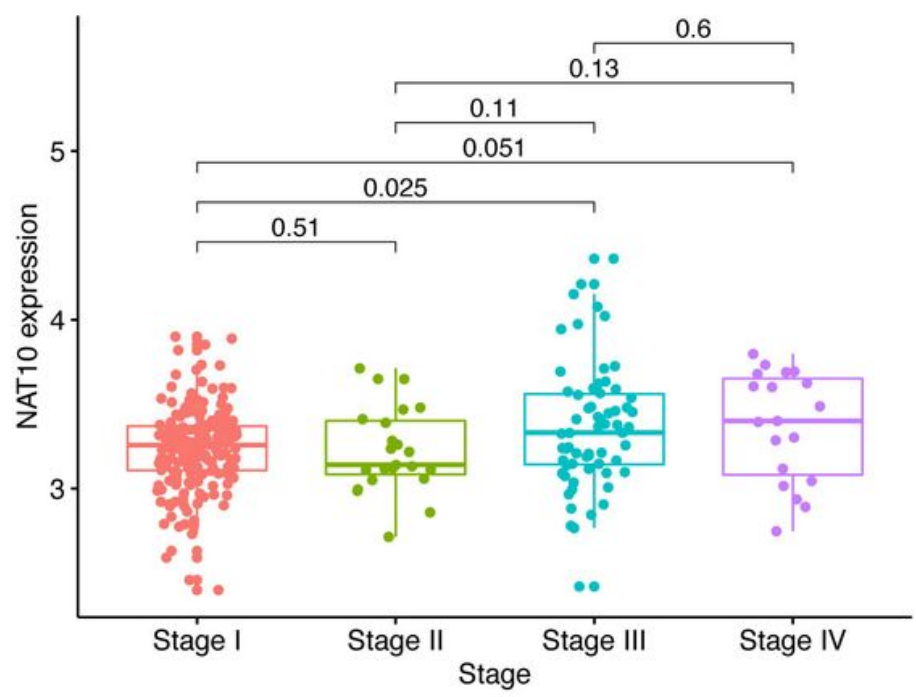

B Cancer: HNSC

Stage 审 Stage I 审 Stage II 审 Stage III 宊 Stage IV

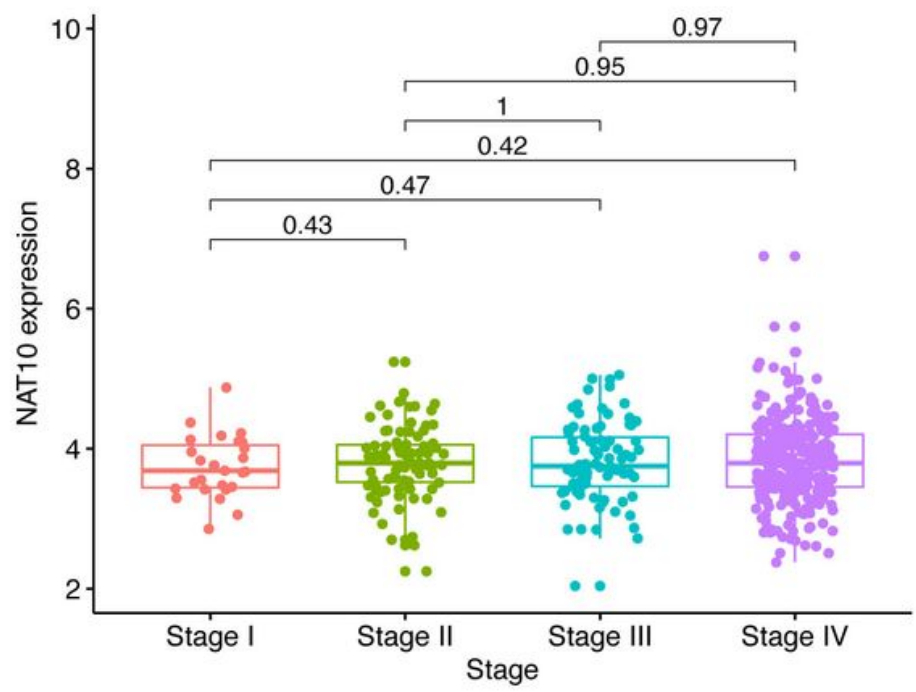

$\mathbf{D}_{\text {Cancer: LIHC }}$

Stage

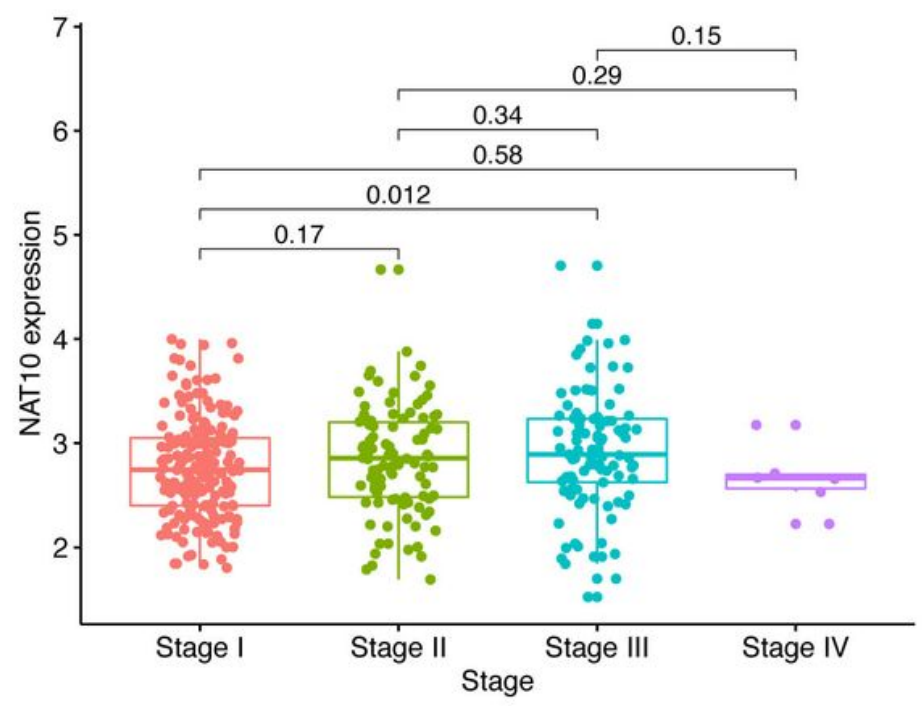

Figure 4

Correlation of NAT10 expression levels with stage in ACC, HNSC, KIRP, and LIHC. Relationships of NAT10 expression with stage in ACC (A), HNSC (B), KIRP (C), and LIHC (D). 

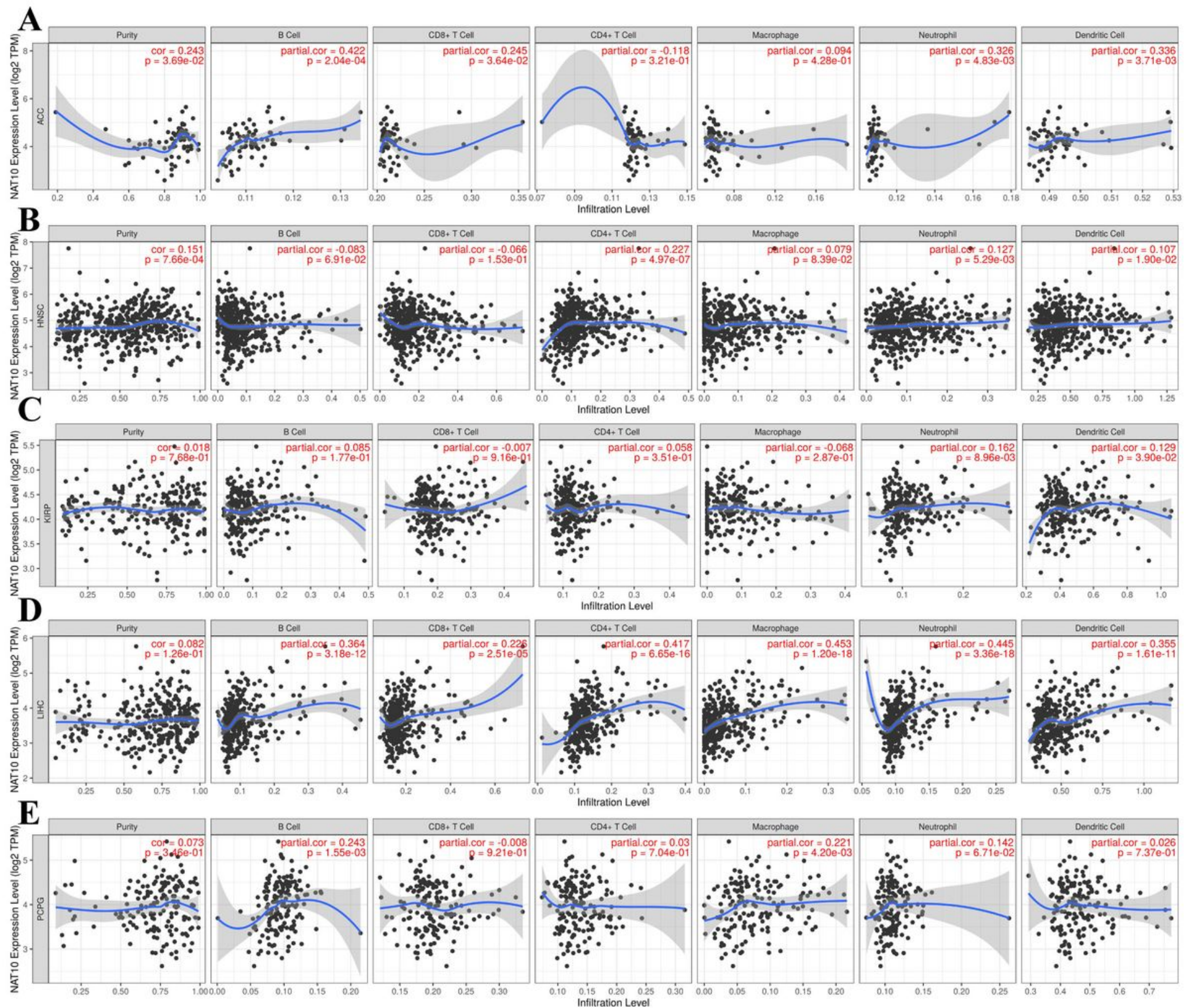

Figure 5

Correlation of NAT10 expression with immune infiltration levels in ACC, HNSC, KIRP, LIHC, and PCPG. (A) Correlations of NAT10 expression with immune infiltration levels in ACC (A), HNSC (B), KIRP (C), LIHC (D), and PCPG (E). 

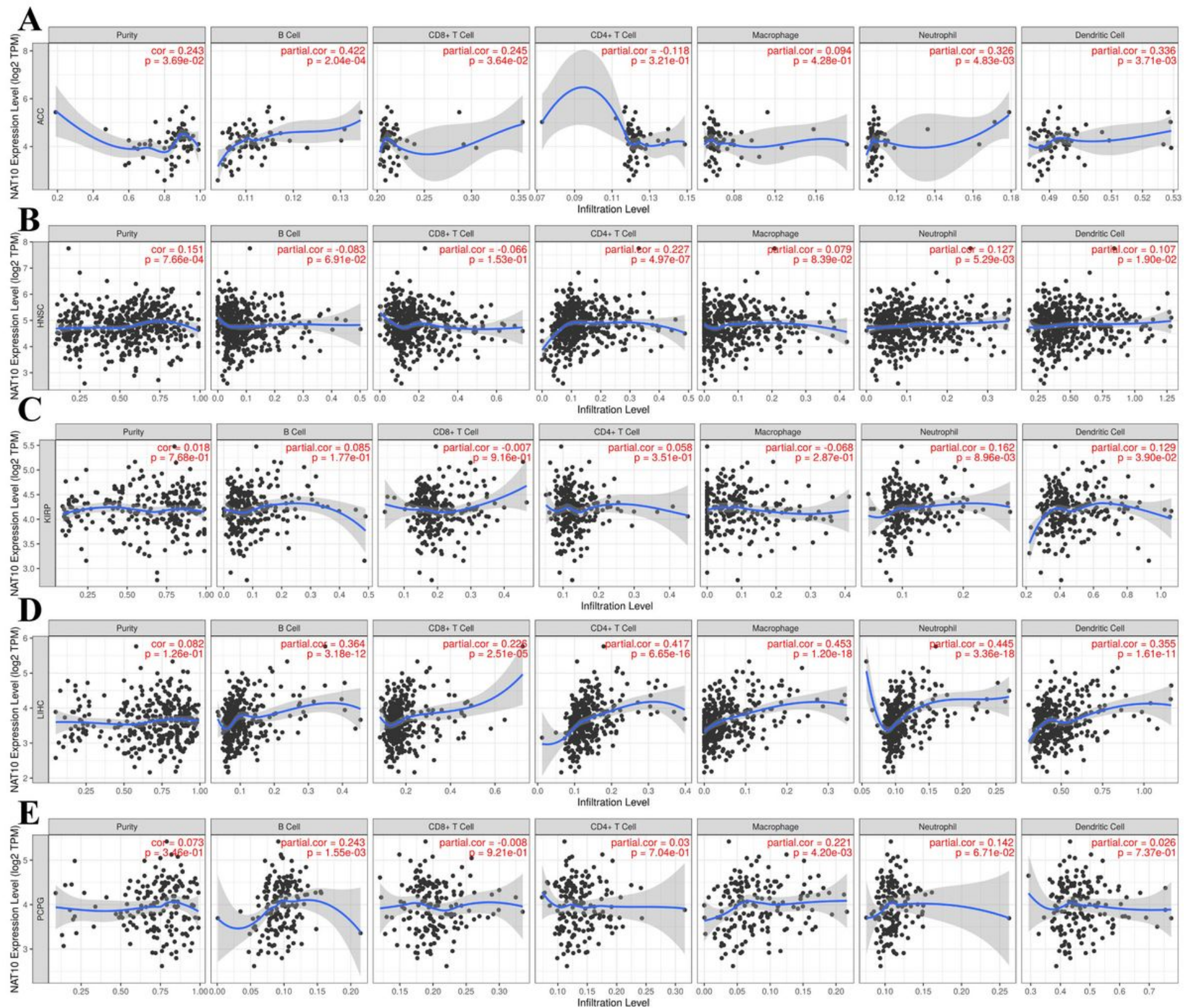

Figure 5

Correlation of NAT10 expression with immune infiltration levels in ACC, HNSC, KIRP, LIHC, and PCPG. (A) Correlations of NAT10 expression with immune infiltration levels in ACC (A), HNSC (B), KIRP (C), LIHC (D), and PCPG (E). 

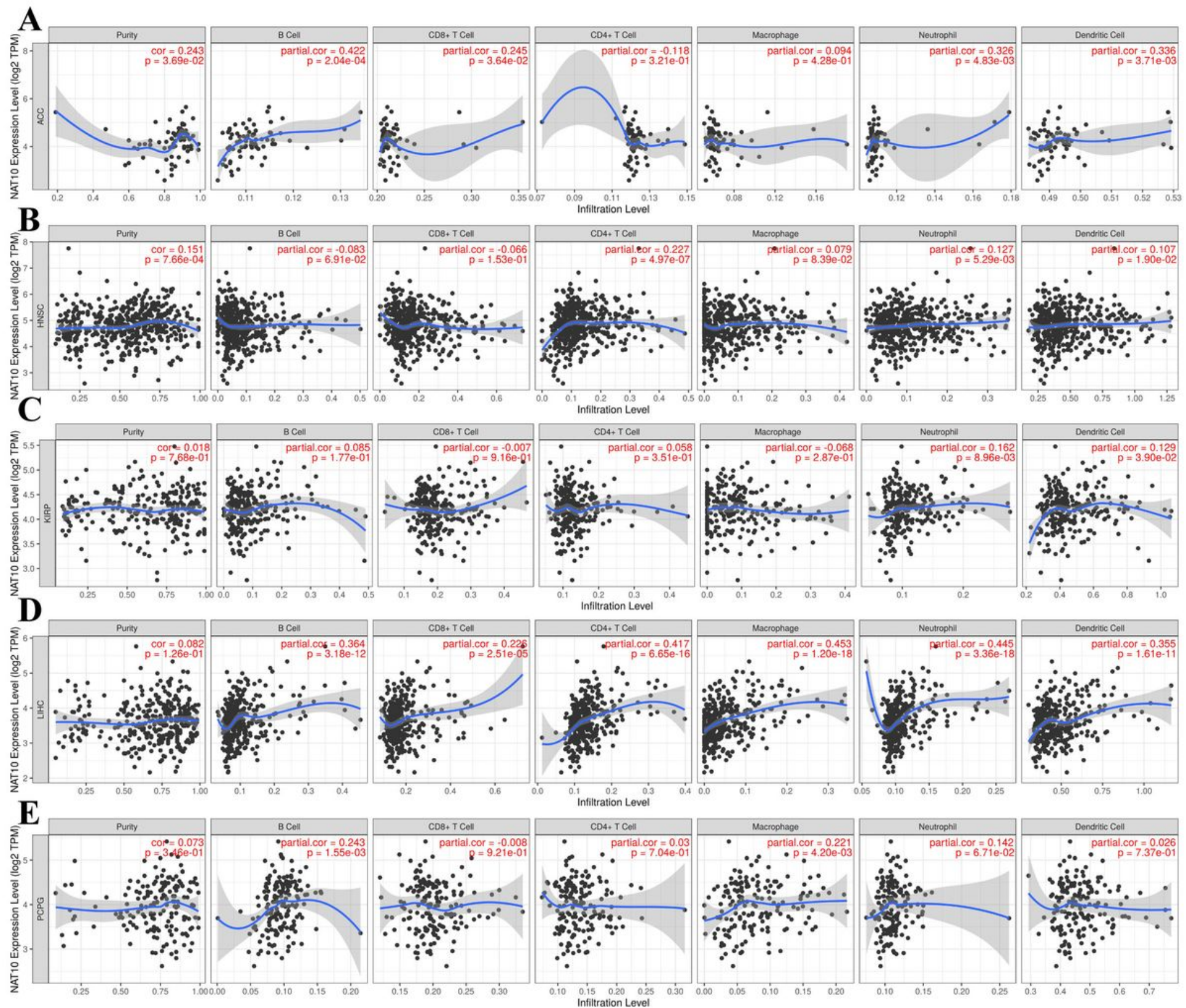

Figure 5

Correlation of NAT10 expression with immune infiltration levels in ACC, HNSC, KIRP, LIHC, and PCPG. (A) Correlations of NAT10 expression with immune infiltration levels in ACC (A), HNSC (B), KIRP (C), LIHC (D), and PCPG (E). 


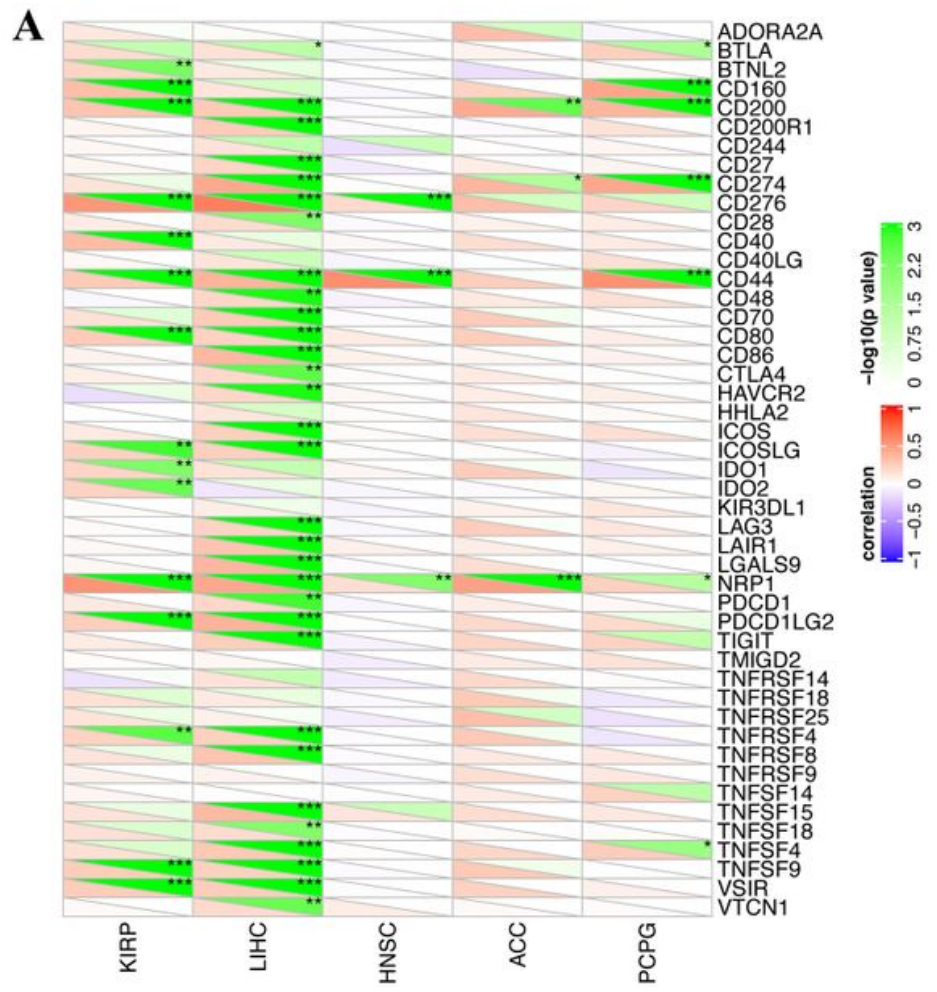

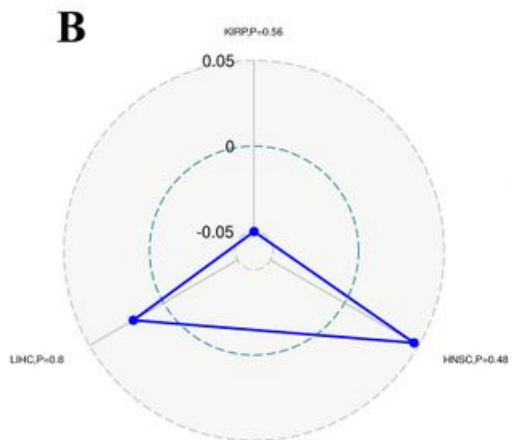

Immune neoantigen

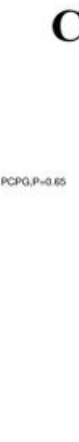

C

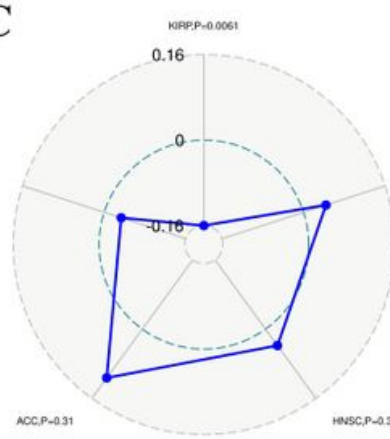

TMB

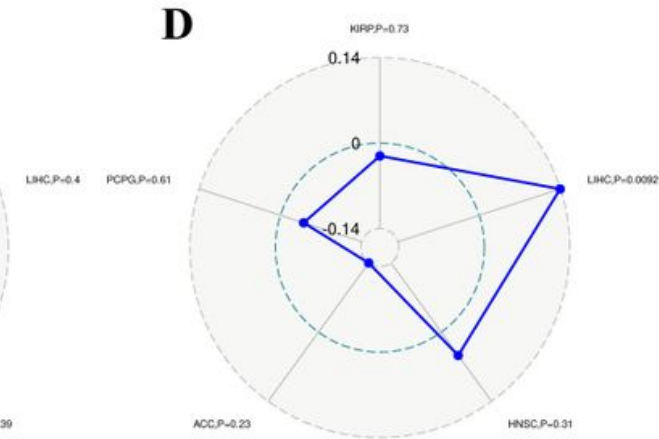

MSI

\section{Figure 6}

Relationship between NAT10 expression and immune checkpoint gene expression, immune neoantigens, TMB, and MSI in ACC, HNSC, KIRP, LIHC, and PCPG. (A) Correlations of NAT10 expression with immune checkpoint gene expression. The lower triangle in each tile indicates coefficients calculated by Pearson's correlation test, and the upper triangle indicates the log10-transformed P-value. (B) Correlations between immune neoantigens and NAT10 expression. (C) Correlations between TMB and NAT10 expression. (D) Correlations between MSI and NAT10 expression. ${ }^{*} P<0.05,{ }^{\star *} P<0.01,{ }^{\star \star *} P<0.001$. 


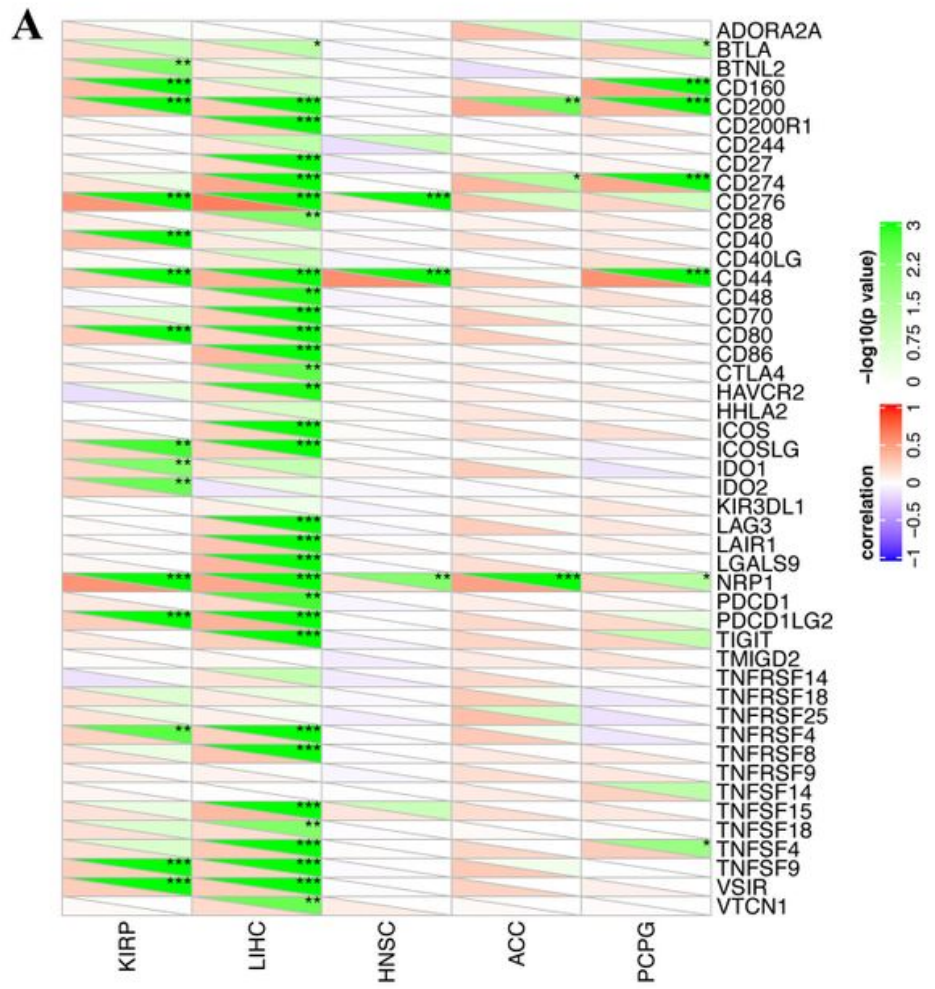

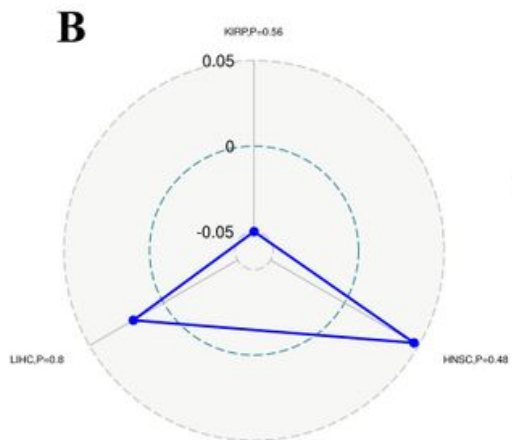

Immune neoantigen

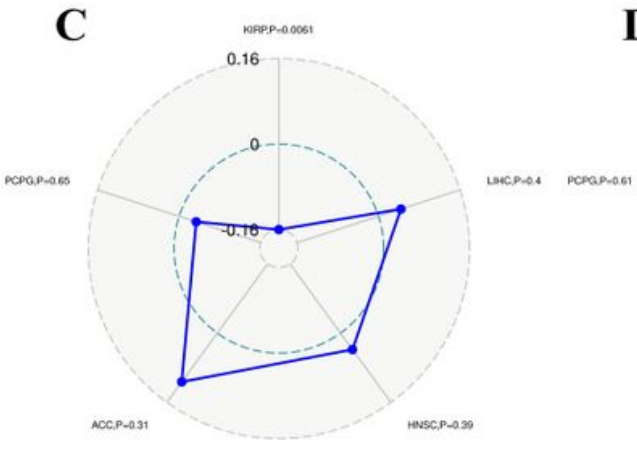

TMB

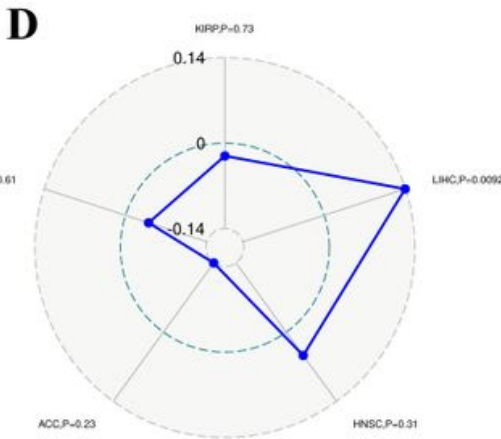

MSI

\section{Figure 6}

Relationship between NAT10 expression and immune checkpoint gene expression, immune neoantigens, TMB, and MSI in ACC, HNSC, KIRP, LIHC, and PCPG. (A) Correlations of NAT10 expression with immune checkpoint gene expression. The lower triangle in each tile indicates coefficients calculated by Pearson's correlation test, and the upper triangle indicates the log10-transformed P-value. (B) Correlations between immune neoantigens and NAT10 expression. (C) Correlations between TMB and NAT10 expression. (D) Correlations between MSI and NAT10 expression. ${ }^{*} P<0.05,{ }^{\star *} \mathrm{P}<0.01,{ }^{\star \star *} \mathrm{P}<0.001$. 


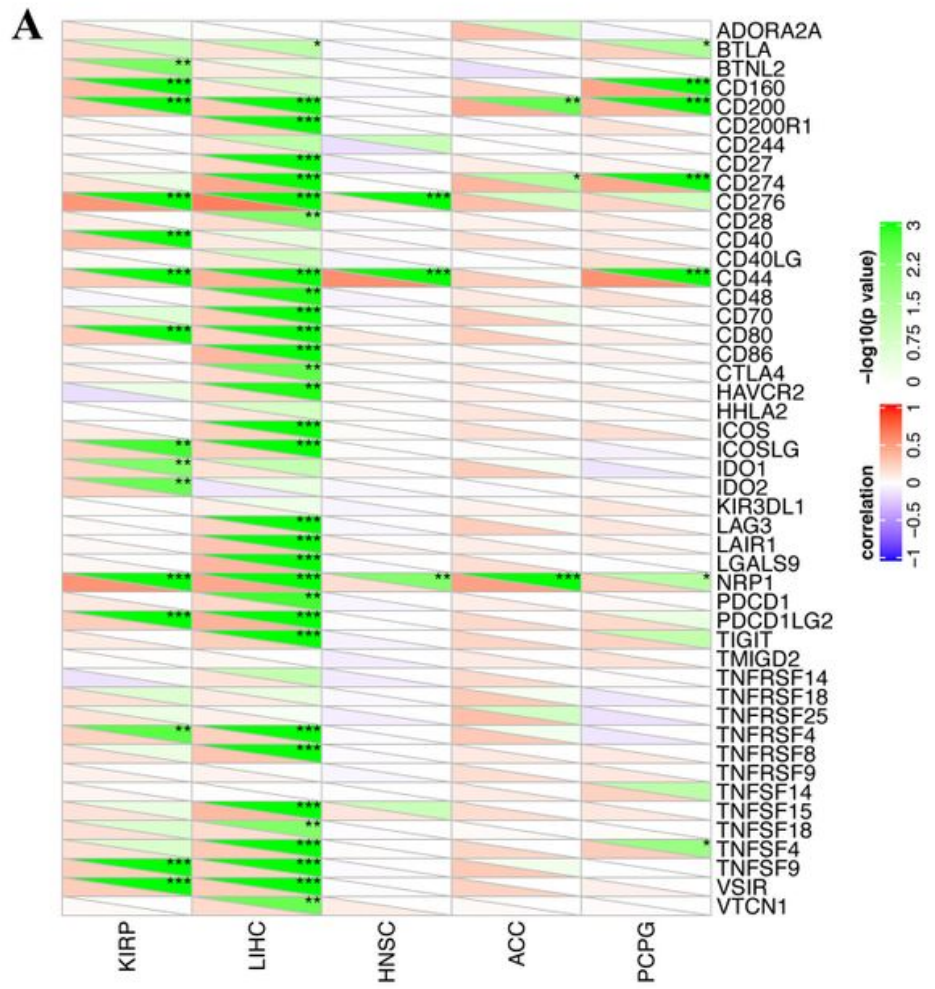

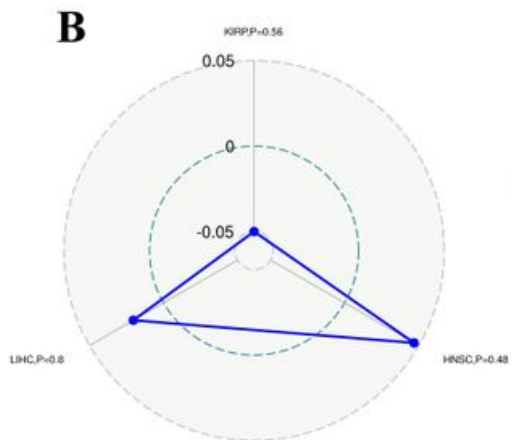

Immune neoantigen

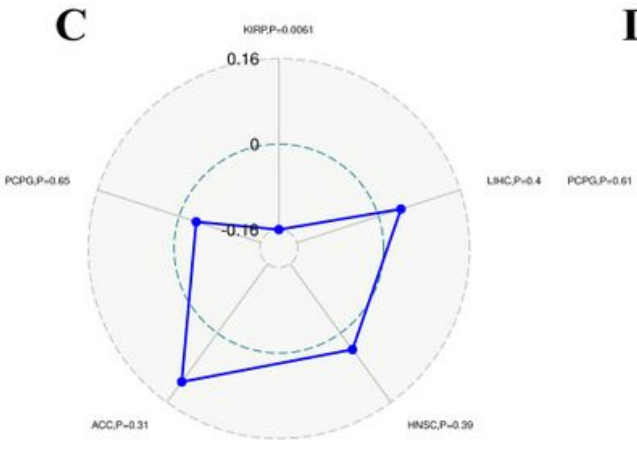

TMB

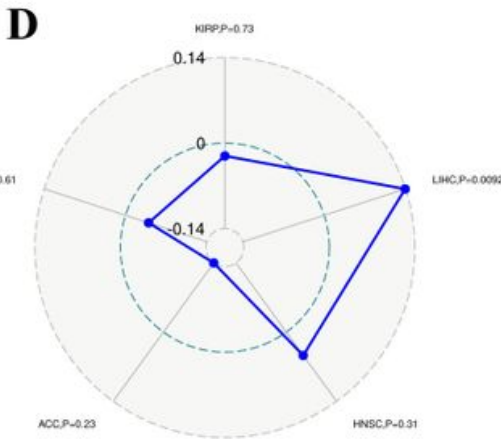

MSI

\section{Figure 6}

Relationship between NAT10 expression and immune checkpoint gene expression, immune neoantigens, TMB, and MSI in ACC, HNSC, KIRP, LIHC, and PCPG. (A) Correlations of NAT10 expression with immune checkpoint gene expression. The lower triangle in each tile indicates coefficients calculated by Pearson's correlation test, and the upper triangle indicates the log10-transformed P-value. (B) Correlations between immune neoantigens and NAT10 expression. (C) Correlations between TMB and NAT10 expression. (D) Correlations between MSI and NAT10 expression. ${ }^{*} P<0.05,{ }^{\star *} \mathrm{P}<0.01,{ }^{\star \star *} \mathrm{P}<0.001$. 


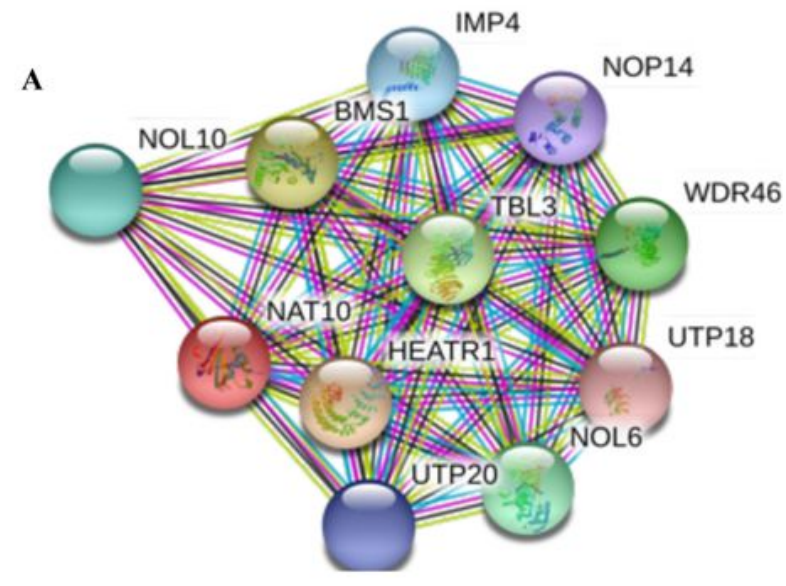

Known Interactions
- -5 from curated databases

Predicted Interactions

-S-O geneneighborhood

C-O genefusions

- - gene co-occurrence

Others

○-O textmining

- - co-expression

- -9 protein homology

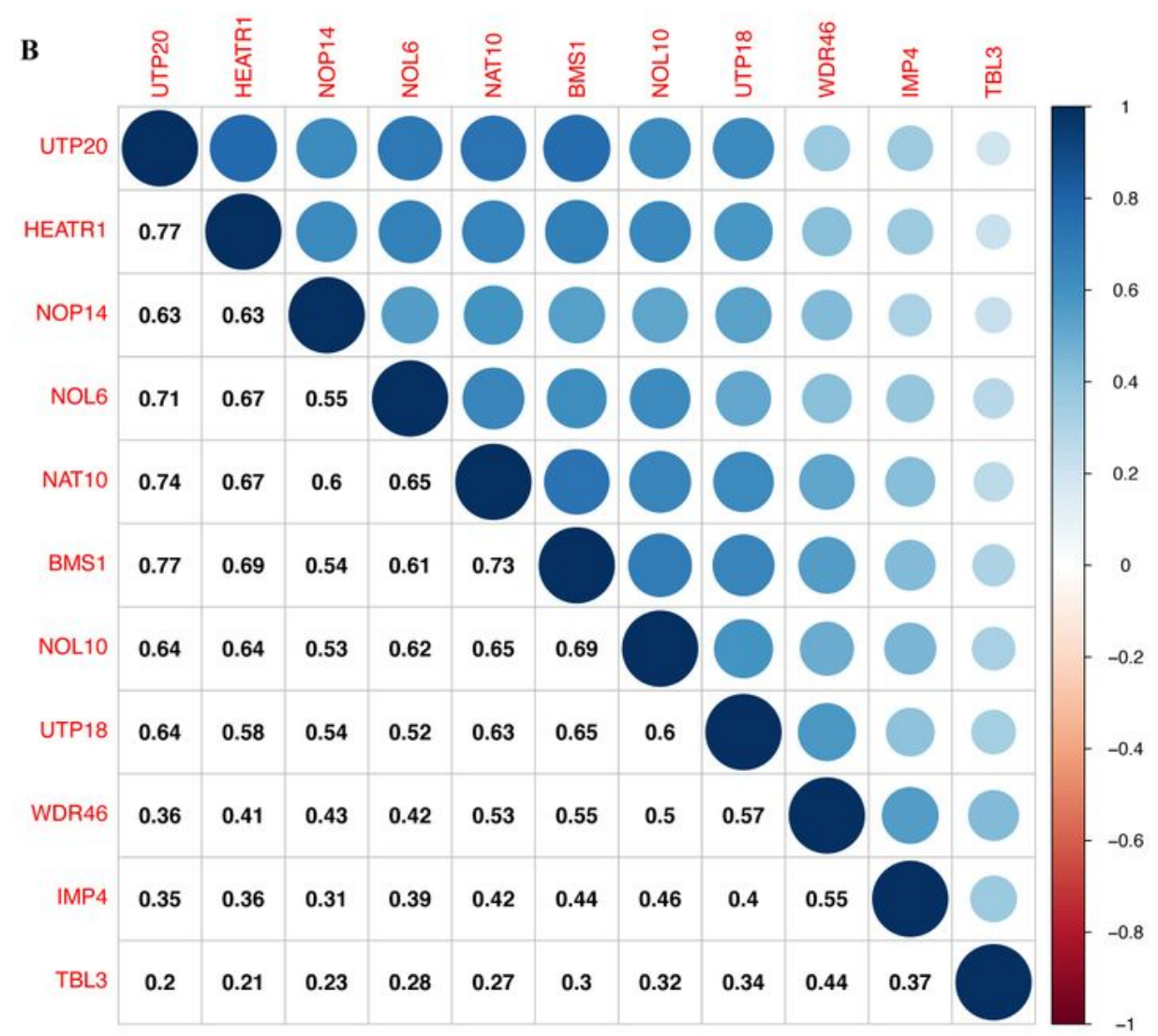

Figure 7

Potential NAT10-interacting proteins and correlations among them. (A) PPI network constructed using the STRING database. (B) Pearson correlation analysis was used to determine the correlations among NAT10 and 10 potential interacting proteins. 


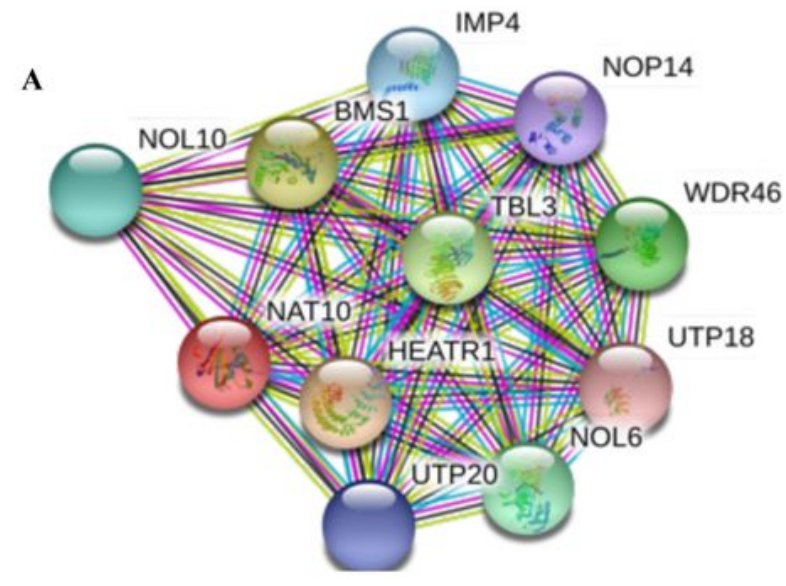

Known Interactions
- -5 from curated databases

Predicted Interactions

-S-O geneneighborhood

C-O genefusions

- - gene co-occurrence

Others

○-O textmining

- - co-expression

- -9 protein homology

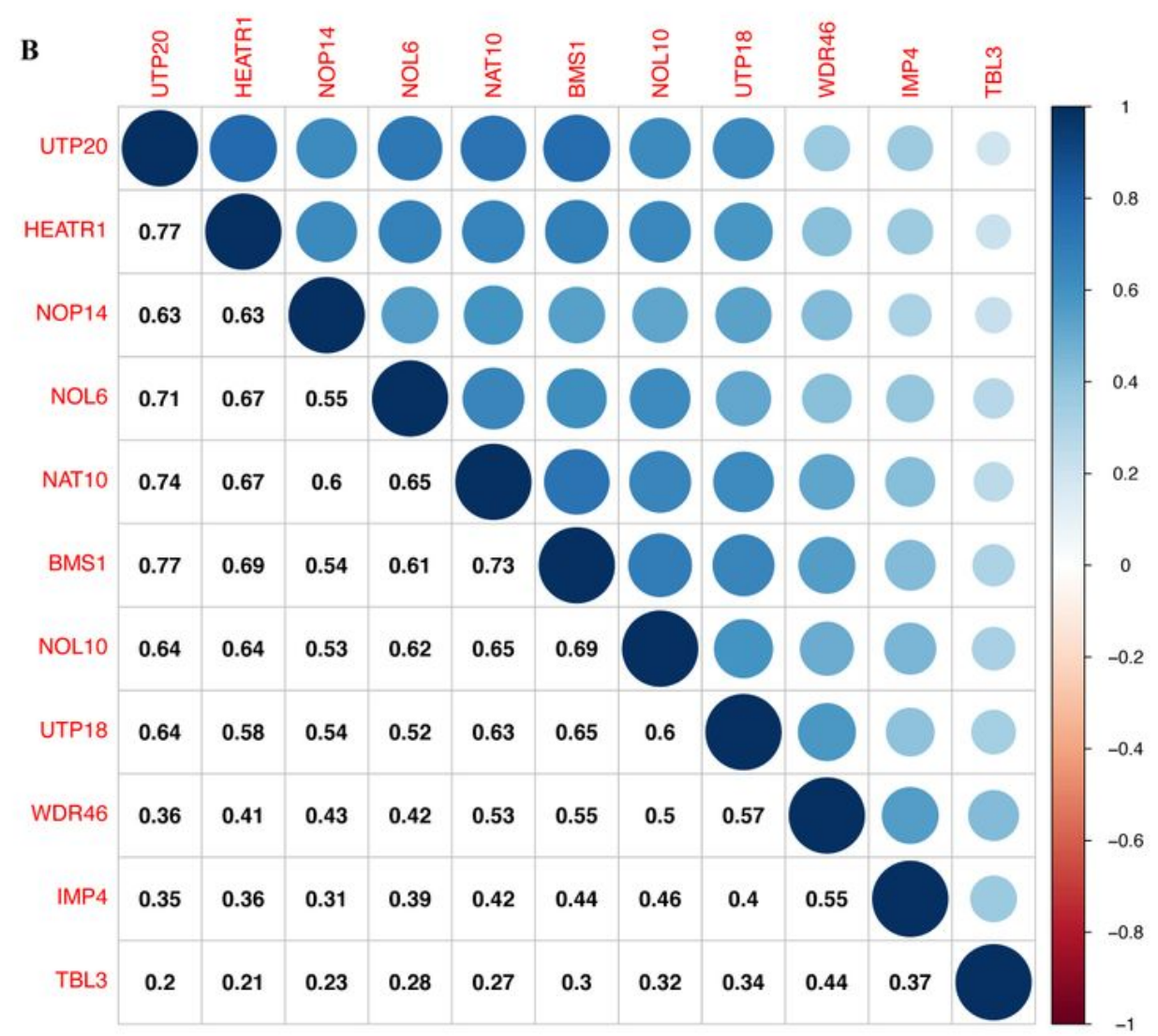

Figure 7

Potential NAT10-interacting proteins and correlations among them. (A) PPI network constructed using the STRING database. (B) Pearson correlation analysis was used to determine the correlations among NAT10 and 10 potential interacting proteins. 


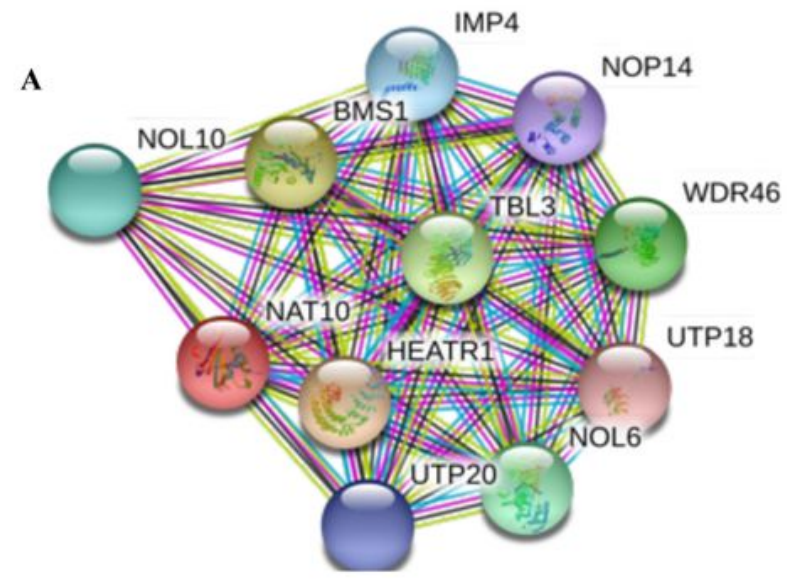

Known Interactions
- -5 from curated databases

Predicted Interactions

-S-O geneneighborhood

C-O genefusions

- - gene co-occurrence

Others

○-O textmining

- - co-expression

- -9 protein homology

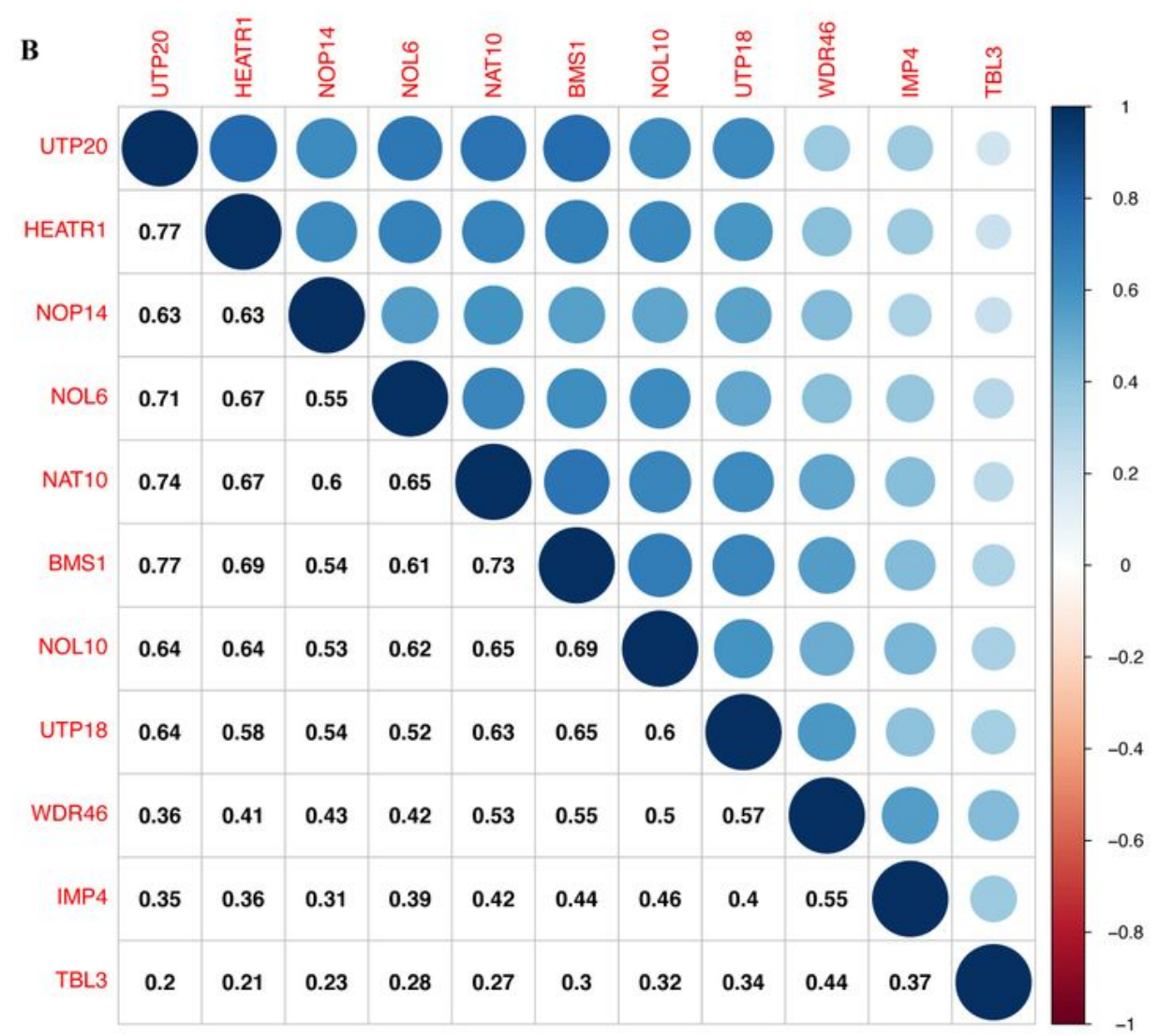

Figure 7

Potential NAT10-interacting proteins and correlations among them. (A) PPI network constructed using the STRING database. (B) Pearson correlation analysis was used to determine the correlations among NAT10 and 10 potential interacting proteins.

\section{Supplementary Files}


This is a list of supplementary files associated with this preprint. Click to download.

- Table1.docx

- Table1.docx

- Table1.docx

- Supplementaryfigure2.pdf

- Supplementaryfigure2.pdf

- Supplementaryfigure2.pdf

- supplementfigure1.pdf

- supplementfigure1.pdf

- supplementfigure1.pdf

- SupplementaryTable5.docx

- SupplementaryTable5.docx

- SupplementaryTable5.docx

- Supplementarytable4.docx

- Supplementarytable4.docx

- Supplementarytable4.docx

- Supplementarytable3.docx

- Supplementarytable3.docx

- Supplementarytable3.docx

- Supplementarytable2.docx

- Supplementarytable2.docx

- Supplementarytable2.docx

- Supplementarytable1.docx

- Supplementarytable1.docx

- Supplementarytable1.docx 